\title{
Biomarkers for sepsis: more than just fever and leukocytosis-a narrative review
}

\author{
Tatiana Barichello ${ }^{1,2^{*}} \mathbb{0}$, Jaqueline S. Generoso ${ }^{1}$, Mervyn Singer ${ }^{3}$ and Felipe Dal-Pizzol ${ }^{1}$
}

\begin{abstract}
A biomarker describes a measurable indicator of a patient's clinical condition that can be measured accurately and reproducibly. Biomarkers offer utility for diagnosis, prognosis, early disease recognition, risk stratification, appropriate treatment (theranostics), and trial enrichment for patients with sepsis or suspected sepsis. In this narrative review, we aim to answer the question, "Do biomarkers in patients with sepsis or septic shock predict mortality, multiple organ dysfunction syndrome (MODS), or organ dysfunction?" We also discuss the role of pro- and anti-inflammatory biomarkers and biomarkers associated with intestinal permeability, endothelial injury, organ dysfunction, blood-brain barrier (BBB) breakdown, brain injury, and short and long-term mortality. For sepsis, a range of biomarkers is identified, including fluid phase pattern recognition molecules (PRMs), complement system, cytokines, chemokines, damageassociated molecular patterns (DAMPs), non-coding RNAs, miRNAs, cell membrane receptors, cell proteins, metabolites, and soluble receptors. We also provide an overview of immune response biomarkers that can help identify or differentiate between systemic inflammatory response syndrome (SIRS), sepsis, septic shock, and sepsis-associated encephalopathy. However, significant work is needed to identify the optimal combinations of biomarkers that can augment diagnosis, treatment, and good patient outcomes.
\end{abstract}

Keywords: Biomarker, Systemic inflammatory response, Sepsis, Septic shock, Sepsis-associated encephalopathy

\section{Introduction}

A biomarker describes a measurable indicator of biological status in normal and pathogenic processes. It may be helpful as a theranostic for identifying suitable patients for therapeutic intervention and titrating the degree and/or duration of intervention. A biomarker should be accurate and reproducible. In the ideal scenario, the biomarker (or combination of biomarkers) should offer both high specificity and sensitivity for diagnosing a condition, but either alone may be adequate as a 'rule-in' or 'ruleout' test.

Sepsis represents a dysregulated immune response to infection that leads to organ dysfunction [1]. Host

\footnotetext{
*Correspondence: Tatiana.Barichello@uth.tmc.edu

2 Faillace Department of Psychiatry and Behavioral Sciences, McGovern

Medical School, The University of Texas Health Science Center at Houston (UTHealth), Houston, TX 77054, USA

Full list of author information is available at the end of the article
}

response biomarkers play a critical role in diagnosis, early recognition of organ dysfunction, risk stratification, prognostication, and patient management, including antibiotic stewardship. Biomarkers may also be helpful for trial enrichment to identify suitable patients and/or risk categorization for an intervention. A wide range of biomarkers, measured by a host of different technologies, are being investigated to discriminate a systemic inflammatory response syndrome (SIRS) rapidly, which is an excessive defensive body's response to a harmful stressor (for example, infection, trauma, surgery, acute inflammation, ischemia or reperfusion, or cancer) [2] or early identification of infection-triggered organ dysfunction (sepsis). Also, the quick sepsis related organ failure assessment (qSOFA) is intended to raise suspicion of sepsis and encourage additional action; although, qSOFA is not a substitute for SIRS [3]. These biomarkers include measurement of acute-phase proteins, original author(s) and the source, provide a link to the Creative Commons licence, and indicate if changes were made. The images or other third party material in this article are included in the article's Creative Commons licence, unless indicated otherwise in a credit line to the material. If material is not included in the article's Creative Commons licence and your intended use is not permitted by statutory regulation or exceeds the permitted use, you will need to obtain permission directly from the copyright holder. To view a copy of this licence, visit http://creativecommons.org/licenses/by/4.0/. The Creative Commons Public Domain Dedication waiver (http://creativeco mmons.org/publicdomain/zero/1.0/) applies to the data made available in this article, unless otherwise stated in a credit line to the data. 
cytokines, chemokines, damage-associated molecular patterns (DAMPs), endothelial cell markers, leukocyte surface markers, non-coding RNAs, miRNA, and soluble receptors, as well as metabolites and alterations in gene expression (transcriptomics). Biomarkers may help stratify septic patients into biological phenotypes, for example, hyperinflammatory versus immunosuppressive. Biomarkers can also be used to identify gut permeability, blood-brain barrier (BBB) permeability, probability of hospital readmission, and longer-term outcomes $[4,5]$.

The causative pathogen replicates and releases its constituents such as endo- and exotoxins, and DNA. These constituents are designated pathogen-associated molecular patterns (PAMPs) [6, 7]. PAMPs are recognized by both pattern-recognition receptors (PRRs) and non-PRRs, which are essential components of the immune system [8, 9]. PRRs include several families, including Toll-like receptors (TLRs), nucleotide-binding oligomerization domain-like receptors (NOD)-like receptors (NLRs), a retinoic acid-inducible gene I (RIGI)-like receptors (RLRs), C-type lectin receptors (CLRs), and intracellular DNA-sensing molecules. Non-PRRs include receptors for advanced glycation end products (RAGE), triggering receptors expressed on myeloid cells (TREM), and G-protein-coupled receptors (GPCRs) [10]. The sensing of PAMPs by immune cell receptors triggers a cascade of signaling pathways that activate multiple transcription factors to promote the production and release of pro- and anti-inflammatory mediators such as acute-phase proteins, cytokines, chemokines, as well as antimicrobial peptides, which are needed to eliminate the invading pathogen [11].

The host immune response and pathogen virulence factors will both trigger cell injury and/or induce cell stress. These results in the release of endogenous molecules (DAMPs), exacerbating the inflammatory response. DAMPs are recognized by the same immune receptors that recognize PAMPs $[12,13]$. Many DAMPs have been identified, with some currently used as inflammatory biomarkers. Examples include proteins and cellular molecules related to nucleic acids, such as heat shock proteins (HSPs), the high mobility group box 1 (HMGB-1), and members of the $S 100$ family $[12,14,15]$. The immune response may induce vascular endothelial damage disrupting tight junctions (T.J.), increasing gut permeability, and potentially facilitating translocation of pathogens and/or their PAMPs from the gut to the bloodstream and lymphatics, thereby amplifying the systemic inflammatory response [16]. In addition, an increase of BBB permeability allows circulating immune cells to enter the brain, triggering or exacerbating glial cell activation [17]. These events could trigger an intense and excessive host response activating coagulation and fibrinolytic systems, activating or suppressing hormonal, bioenergetic, and metabolic pathways, and inducing macro- and microcirculatory changes with a net result of multiple organ dysfunction. In the past few decades, researchers have studied each inflammatory response stage during SIRS, sepsis, and septic shock, metabolites associated with inflammatory cascades, and cellular components that could be used as biomarkers. These biomarkers could help identify endothelial damage, intestinal permeability, organ failure, BBB breakdown and predict rehospitalization, short- and long-term mortality, and cognitive consequences in survivors [18].

For this narrative review, we addressed the question, "Do biomarkers in patients with sepsis or septic shock predict mortality, MODS, or organ dysfunction?" Studies were identified by searching PubMed/MEDLINE (National Library of Medicine) databases for peerreviewed journal articles published until October 2021. The abovementioned databases were searched with the following combinations of keywords: ("inflammatory response syndrome" OR "SIRS" OR "sepsis" OR "septic shock" OR "sepsis-associated encephalopathy" OR "SAE") AND ("markers" OR "biomarkers" OR "biological markers" OR "biological measures" OR "molecular predictor"). We omitted review articles, in vitro studies, and animal studies.

\section{The humoral innate immune response, cytokines, and chemokines}

The humoral innate immune response consists of multiple components, including fluid phase pattern recognition molecules (PRMs) and the complement system. PRMs include C-reactive protein (CRP), serum amyloid P component (SAP), and pentraxin 3 (PTX3) [19]. The rise in CRP level is primarily induced by interleukin (IL)- 6 and IL- $1 \beta$ acting on the gene responsible for CRP transcription during the acute phase of an inflammatory process. CRP is a pentameric acutephase reactant protein whose conformation facilitates the ability to trigger complement activation and activate platelets, monocytes, and endothelial cells [20]. Furthermore, CRP is one of the most widely used and investigated biomarkers [21]. A prospective multicenter cohort study followed 483 adult patients who survived hospitalization for sepsis for up to one year. IL-6, high-sensitivity $C$ reactive protein (hs-CRP), soluble programmed death-ligand 1 (sPD-L1), E-selectin, and intercellular adhesion molecule 1 (ICAM-1) were evaluated at five-time points during and after hospitalization. A comparison was made between a phenotype with hyperinflammation (high levels of IL-6 and hs-CRP) and a phenotype of immunosuppression (high sPD-L1 levels). Compared with a normal phenotype, 
both hyperinflammation and immunosuppression phenotypes had higher 6-month hospital readmission rates and 1-year mortality rates, both all-cause and attributable to cardiovascular or cancer [22].

Pentraxin (PTX-3) is secreted by macrophages, dendritic cells, macrophages, fibroblasts, mesangial cells, and glial cells under pathogen or inflammatory stimuli [19]. Plasma PTX-3 was assessed on days 1, 2, and 7 in 958 patients with sepsis or septic shock included in the Albumin Italian Outcome Sepsis (ALBIOS) study. The researchers assessed a possible association between PTX-3 levels and clinical severity, organ dysfunction, treatment, and mortality within 90 days. PTX-3 levels were elevated at the onset of sepsis and increased with illness severity and the number of organ dysfunctions. PTX-3 levels decreased between days 1 to 7 , but this was less prominent in patients with septic shock [23]. In a prospective observational analysis, PTX-3, IL-6, procalcitonin (PCT), and lactate combined showed excellent performance in predicting 28-day all-cause mortality among patients diagnosed with sepsis or septic shock and superior to the Sequential Organ Failure Assessment (SOFA) score [24].

In a prospective pilot study of markers of complement activation in sepsis, higher $\mathrm{C} 4 \mathrm{~d}$ (3.5-fold), factor $\mathrm{Bb}$ (6.1fold), C3 (0.8-fold), C3a (11.6-fold), and C5a (1.8-fold) levels were seen compared with healthy volunteer controls [25]. In another study of 49 sepsis patients, 34 developed disseminated intravascular coagulation (DIC), and eight died. Patients with DIC had lower C3 levels and higher SC5b-9 levels. On stratifying by SC5b-9 quartile (ng/mL: low: $<260$, moderate: 260-342, high: 343501, highest:>501), coagulation parameters were most deranged in the highest quartile with prolonged thrombocytopenia and higher mortality (33\%) [26].

The activation of PRRs culminates in the stimulation of transcription factors resulting in the expression and secretion of proinflammatory cytokines, including tumor necrosis factor-alpha (TNF- $\alpha$ ), IL-1- $\beta$, IL-6, and interferons (IFNs). These inflammatory mediators are required for host defense against pathogens and activation of the adaptive immune response. A retrospective study evaluated a broad panel of cytokines and found IL-1 $\beta$, IL-6, IL-8, MCP-1, IL-10, and plasminogen activator inhibitor 1 (PAI-1) levels were increased in the acute phase of sepsis in both critically and non-critically ill patients. In addition, levels of IL-10 (days 1, 2, and 4), IL-6 and PAI-1 (days 2 and 4), and IL-8 (day 4) increased in critically ill patients compared to non-critically ill [27]. In summary, hs-CRP, IL-6, and PAI-1 circulatory levels may have utility in stratifying a hyperinflammatory patient phenotype.

\section{DAMPs}

DAMPs are endogenous danger molecules released from damaged or stressed cells. These molecules activate the innate immune system through interaction with PRRs. DAMPs contribute to the host defense but can also promote pathological inflammatory responses. Calprotectin, a protein found in the cytosol of neutrophils and macrophages, is released under cell stress or damage. In a mixed population study, plasma calprotectin levels were higher in sepsis than in trauma patients and other medical conditions. Calprotectin levels were higher in patients who did not survive for 30 days. Plasma PCT did not differ between the groups or as a prognosticator of the outcome. Receiver operating characteristic (ROC) analysis, used as a sepsis biomarker, had a higher area under the curve (AUC) value for calprotectin (AUC: 0.79) compared to PCT (AUC: 0.49) [28].

A prospective study evaluated IL-6, HMGB-1, and neutrophil gelatinase-associated lipocalin (NGAL) in 14 septic patients and 16 patients without sepsis admitted to the ICU. In patients with sepsis, IL-6 decreased levels were associated with ICU survival; NGAL levels rose in non-survivors, while HMGB-1 levels were unchanged in both survivors and non-survivors regardless of complications [29].

\section{Endothelial cells and BBB markers}

The first step in endothelial and BBB injury is the breakdown and destruction of proteins followed by release into the bloodstream. These proteins or peptides can be evaluated as a marker of endothelial cells and BBB integrity [30]. Plasma levels of occludin (OCLN), claudin-5 (CLDN-5), zonula-occludens 1 (ZO-1), PCT, and lactate were assessed in 51 septic patients. OCLN and ZO-1 were elevated with disease severity and positively correlated with the Acute Physiology and Chronic Health Evaluation II (APACHE-II) and SOFA scores and lactate levels. The predictive value for in-hospital mortality of ZO-1 was comparable to that of lactate levels, APACHE-II, and SOFA scores but superior to OCLN and PCT [31]. In a case series of brain autopsies from adults who died from sepsis, $38 \%$ had no OCLN expression in the endothelium of cerebral microvessels. BBB damage was associated with higher maximum SOFA scores and PCT levels $>10 \mu \mathrm{g} / \mathrm{L}$. BBB damage in the cerebellum was more common with CRP values $>100 \mathrm{mg} / \mathrm{L}$ [32]. Soluble fms-like tyrosine kinase 1 (sFlt-1), soluble E-selectin (sE-selectin), soluble intercellular adhesion molecule 1 (sICAM-1), soluble vascular cell adhesion molecule 1 (sVCAM-1), and PAI-1 were evaluated in another studies. All these evaluated endothelial biomarkers were associated with sepsis severity. sFlt-1 had the strongest association with the SOFA score, while sFlt-1 and PAI-1 
had the highest area under the operating receiver characteristic curve for mortality [33].

Syndecan-1 is a structural component of the endothelium. Antithrombin, PAI-1, syndecan-1, VCAM-1, E-selectin, IL-1 $\beta$, IL-6, IL-8, HMGB-1, and histone-H3 were increased in septic patients compared with healthy controls. Non-survivors had a higher syndecan-1 level compared with survivors. On day one, an association was seen between syndecan-1 levels and APACHE-II, SOFA, DIC scores, hemostatic markers, IL-1 $\beta$, IL-8, and PAI-1. Day 1 syndecan-1 levels were also significantly higher in patients with DIC and had reliable discriminative power to predict both DIC development and subsequent mortality [34].

The serum biomarker, calcium-binding protein $B$ (S100B), reflects BBB disruption, glial cell injury, and activation. S100B is used to evaluate brain injury severity and predict outcomes from stroke, traumatic brain injury, encephalopathy, and delirium [35]. A prospective cohort study demonstrated that day three values for predicting 180-day mortality were superior to day one $(0.731$ vs. 0.611) [36]. Patients with sepsis-associated encephalopathy also had elevated levels. In another observational study of 22 patients with septic shock, delirium was present in ten. The odds ratio for the risk of developing delirium with an $\mathrm{S} 100 \mathrm{~B}>0.15 \mu \mathrm{g} / \mathrm{L}$ was 18.0. Patients with delirium had higher plasma levels of IL-6. S100B and IL-6 levels were positively correlated [37]. S100B, PAI1 , angiopoietin (Ang)-2, ZO-1, and OCLN are the main biomarkers currently used to evaluate the vascular injury and BBB permeability.

\section{Gut permeability markers}

Critically ill patients show an increase in gut permeability, which may trigger a systemic inflammatory response syndrome and multiple organ dysfunction syndromes (MODS) [38]. Plasma zonulin levels were higher in sepsis patients compared to a post-surgical control group or healthy volunteers [39]. In another study, serum levels of intestinal fatty acid-binding protein (I-FABP) were higher in patients with sepsis and higher still in those with septic shock. Serum D-lactic acid levels were also elevated with sepsis but did not differentiate severity. Neither I-FABP nor D-lactic acid could prognosticate [40].

\section{Non-coding RNAs and miRNA}

A non-coding RNA (ncRNA) is an RNA molecule transcribed from DNA but not translated into proteins. A microRNA is a small non-coding RNA molecule that functions in RNA silencing and post-transcriptional gene expression regulation. ncRNAs and mRNAs are being studied as sepsis biomarkers. For example, long non-coding metastasis-associated lung adenocarcinoma transcript 1 (lnc-MALAT1) and micro RNA (miR)-125a were increased in sepsis patients compared with healthy controls and positively correlated with APACHE-II score, SOFA score, serum creatinine, CRP, TNF- $\alpha$, IL- $1 \beta$, IL- 6 , and IL-8. The lnc-MALAT1/miR-125a axis was also a predictor of increased 28-day mortality risk [41]. In another study lnc-MALAT1 expression was increased in acute respiratory distress syndrome (ARDS) patients compared to non-ARDS patients (AUROC: 0.674). Nonsurvivors compared to survivors (AUROC: 0.651 ) and positively correlated with APACHE-II and SOFA scores, CRP, PCT, TNF- $\alpha$, IL-1 $\beta$, IL-6, and IL-17 [42]. Long noncoding RNA maternally expressed gene 3 (lnc-MEG3), and the lnc-MEG3/miR-21 axis were increased, while miR-21 expression was decreased in sepsis patients compared with healthy controls. lnc-MEG3 (AUROC: 0.887) and the lnc-MEG3/miR-21 ratio (AUROC: 0.934) had high values for predicting elevated sepsis risk, while miR21 (AUROC: 0.801) gave excellent predictive value for a reduced sepsis risk [43]. A further study showed miR$125 \mathrm{a}$ and miR-125b expressions were elevated in sepsis patients compared with healthy controls and were predictive of sepsis risk-miR-125a (AUROC: 0.749) and miR-125b (AUROC: 0.839). No correlation was seen between miR-125a and CRP, TNF- $\alpha$, IL-6, IL-17, and IL-23 in however, miR-125b was positively associated with these cytokines. miR-125a failed to predict 28-day mortality risk (AUROC: 0.588 ) in sepsis patients, whereas miR-125b was superior (AUROC: 0.699) [44].

\section{Membrane receptors, cell proteins, and metabolites}

Cell surface receptors are receptors incorporated into the plasma membrane of cells and act on cell signaling by receiving or binding to extracellular molecules. After detecting such molecules, the production of metabolites occurs. In one study, the cluster of differentiation (CD)13, CD14, CD25, CD64, and human leukocyte antigen (HLA-DR) showed acceptable sensitivity and specificity for mortality prediction (CD13 AUROC:0.824; CD64 0.843; and HLA-DR 0.804) while CD14 and CD25 did not predict mortality [45]. nCD64 expression, in a further study, nCD64, PCT, CRP, and SOFA scores were higher in septic patients, with nCD64 having the highest AUC (0.879) for differentiating a positive microbial culture. This was superior to PCT (0.868), SOFA score (0.701), CRP (0.609), and white blood cell (WBC) count. In predicting 28-day mortality, the combination of $\mathrm{nCD} 64$ and SOFA score had an AUROC of 0.91 versus 0.882 for the combination of PCT and SOFA [46].

A meta-analysis of 19 studies enrolling 3012 patients evaluated the value of PCT (AUROC 0.84) and presepsin (0.87 AUROC) for diagnosing sepsis. The pooled 
sensitivities and specificities were 0.80 and 0.75 for PCT and 0.84 and 0.73 for presepsin [47]. In one study, levels of presepsin, PCT, CRP, and WBC were higher in sepsis patients than in a SIRS group with AUROC values of 0.954 (presepsin), 0.874 (PCT), 0.859 (CRP), and 0.723 (WBC). The cut-off of presepsin for discriminating between sepsis and SIRS was $407 \mathrm{pg} / \mathrm{ml}$, with sensitivity and specificity values of $98.6 \%$ and $82.6 \%$, respectively [48]. In a study of septic children, TREM-1 levels were higher in septic shock patients [49].

\section{Hormones and peptide precursors}

Adrenomedullin (ADM) is synthesized in different tissues, including the adrenal cortex, kidney, lung, blood vessels, and heart. It has biological properties, including vasodilating, inotropic, diuretic, natriuretic, and bronchodilating. In one study, mid-regional pro adrenomedullin (MR-proADM) was an independent predictor of five different organ failures (respiratory, coagulation, cardiovascular, neurological, and renal), compared to lactate which predicted three (coagulation, cardiovascular and neurological), PCT two (cardiovascular and renal) and CRP (none) [50]. MR-proADM most accurately identified patients with a high likelihood of further disease progression compared to other biomarkers and clinical scores [51]. A total of 1089 individuals with either sepsis (142) or septic shock (977) were included in a randomized controlled study. The MR-proADM level within the first $24 \mathrm{~h}$ after sepsis diagnosis was associated with 7-day mortality (AUROC 0.72 and $p<0.001$ ) and 90-day mortality (AUROC 0.71 and $p<0.001$ ). Patients with declining PCT levels but persistently high MR-proADM levels on day-1 or day-4 had a substantially higher mortality risk of 19.1 (8.0-45.9) and 43.1 (10.1-184.0), respectively [52]. Adult patients hospitalized to ICU had their bioactive-ADM levels measured in this retrospective observational study. This study comprised a total of 1867 patients, 632 septic patients, and 267 septic shock patients. The median bioactive-ADM was $74 \mathrm{pg} / \mathrm{mL}$ in sepsis patients, $107 \mathrm{pg} /$ $\mathrm{mL}$ in septic shock, and $29 \mathrm{pg} / \mathrm{mL}$ in non-septic patients. The association of elevated bioactive-ADM and mortality in sepsis patients and the ICU population resulted in O.R.s of 1.23 and 1.22, respectively [53]. In addition, the MR-proADM is potentially removal by continuous renal replacement therapy (CRRT) [54].

N-terminal (N.T.)-prohormone BNP (NT-proBNP) is a non-active prohormone produced by the heart and released in response to changes in intracardiac pressure. Higher levels of NT-proBNP at $24 \mathrm{~h}$ after sepsis onset were associated with lower short physical performance battery (SPPB) scores at 12 months and lower handgrip strength at 6-month and 12-month follow-up. NT-proBNP levels during the acute phase of sepsis may thus be a valuable indicator of a greater risk of long-term impairment in physical function and muscle strength in sepsis survivors [55]. In another study, NT-proBNP levels on admission were higher in-hospital non-survivors $(7908 \mathrm{pg} / \mathrm{mL})$ compared with survivors $(3479 \mathrm{pg} / \mathrm{mL})$. AUROC curves of admission and 72-h NT-proBNP levels for hospital mortality were 0.631 and 0.648 , respectively [56].

PCT is produced in the thyroid's $C$ cells and converted to calcitonin, with no PCT released into the bloodstream. During inflammatory processes, PCT is produced directly by stimulating bacterial components or induced by various inflammatory mediators such as IL-6 and TNF- $\alpha$. PCT and presepsin had similar performance in predicting positive sepsis results with AUROC values of 0.75 and 0.73 , respectively [57]. Another investigation gave AUROC values of 0.87 for PCT and 0.78 for presepsin in predicting bacteremia [58]. Plasma levels of presepsin and PCT were progressively higher in sepsis and septic shock than in non-septic patients. Presepsin was superior to PCT in diagnosing severe communityacquired pneumonia [59], while PCT was marginally superior in another study of septic patients admitted to intensive care [60]. On the other hand, MR-proADM had a better predictive value for septic shock. This study concluded that PCT, MR-proADM, and presepsin are complementary biomarkers that could have utility in the management of septic patients. In an intention-to-treat study comparing PCT versus no PCT guidance, there was no significant difference in 28 -day mortality $(25.6 \%$ PCT versus $28.2 \%$ no PCT, $p=0.34$ ). The use of procalcitonin did not impact investment decisions as measured by the frequency of therapeutic and diagnostic interventions. [61].

\section{Neutrophil-related biomarkers}

High levels of resistin collected on day 1 of ICU admission were associated with an increased likelihood of developing new organ failure, whereas high myeloperoxidase (MPO) levels on day one were associated with an increased risk of developing incident organ failure for clotting and kidney systems [62].

\section{Soluble receptors}

Soluble trigger receptor expressed in the myeloid cell-1 (sTREM-1) is a TREM family member. This receptor offers excellent potential as a biomarker for infectious diseases as it can be measured in different biological fluids, including serum, pleural fluid, sputum, and urine [63]. However, a meta-analysis of 2418 patients enrolled in 19 studies showed serum sTREM-1 had only moderate accuracy in diagnosing patients with suspected sepsis [63]. Combining sTREM-1 with clinical variables offered more significant 
Table 1 Different roles of the biomarkers in sepsis

\begin{tabular}{|c|c|c|}
\hline Biomarker & Function & References \\
\hline \multicolumn{3}{|c|}{ Acute-phase proteins } \\
\hline \multirow[t]{3}{*}{ CRP, hsCRP } & Response to infection and other inflammatory stimuli & {$[4,69,70]$} \\
\hline & Predictive for increased 28-day mortality in patients with sepsis & \\
\hline & Hyperinflammatory phenotype & \\
\hline Complement & Prognosis of disease severity & {$[25,71]$} \\
\hline Proteins & C5a can be predictive for DIC & \\
\hline \multirow[t]{3}{*}{ PTX-3 } & Discrimination of sepsis and septic shock & {$[72,73]$} \\
\hline & Diagnosis of sepsis and septic shock during the first week in the ICU & \\
\hline & Prediction of septic shock & \\
\hline \multicolumn{3}{|c|}{ Cytokines and chemokines } \\
\hline IL-10 & Hypoinflammatory phenotype & {$[22,71]$} \\
\hline \multirow[t]{2}{*}{ MCP-1 } & It differentiates patients with septic shock from patients with sepsis & {$[73,74]$} \\
\hline & Mortality prognosis at 30 days and six months & \\
\hline \multirow[t]{4}{*}{ TNF-a, IL-1ß, IL-6 } & IL-6 all-cause mortality prognosis at 30 days and six months & {$[27,74]$} \\
\hline & $\mathrm{IL}-1 \beta$ and IL- 6 acute phase of sepsis & \\
\hline & It was increased in the hyperinflammatory phenotype & \\
\hline & Organ dysfunction prognosis & \\
\hline \multicolumn{3}{|l|}{ DAMPs } \\
\hline \multirow[t]{2}{*}{ Calprotectin } & PCT to distinguish between patients with sepsis and patients without sepsis in the ICU & [28] \\
\hline & Predictive for 30-day mortality & \\
\hline HMGB-1 & Worst prognosis and higher 28-day mortality & {$[75,76]$} \\
\hline \multicolumn{3}{|c|}{ Endothelial cells and BBB markers } \\
\hline \multirow[t]{2}{*}{ Syndecan-1 } & Increase related to sepsis severity & {$[34]$} \\
\hline & Discriminative power for DIC and subsequent mortality & \\
\hline \multirow[t]{2}{*}{ VLA-3 (а3ß1) } & Indicative of sepsis & {$[77,78]$} \\
\hline & Discrimination of sepsis and SIRS & \\
\hline \multirow[t]{3}{*}{ Ang-1 } & $\begin{array}{l}\text { It stabilizes the endothelium and inhibits vascular leakage by constitutively activating } \\
\text { the Tie-2 receptor }\end{array}$ & [79] \\
\hline & $\begin{array}{l}\text { Ang-2/Ang-1, Ang- } 1 / \text { Tie-2 ratio has a prognosis for } 90 \text {-day mortality in sepsis and } \\
\text { septic shock in the ICU higher than the PCT and SOFA score }\end{array}$ & \\
\hline & Independent and effective predictors of SOFA score changes & \\
\hline \multirow[t]{2}{*}{ Ang-2 } & $\begin{array}{l}\text { It can disrupt microvascular integrity by blocking the Tie-2 receptor, which results in } \\
\text { vascular leakage }\end{array}$ & {$[73,79]$} \\
\hline & Individuals with septic shock had higher levels of Ang-2 than those with sepsis & \\
\hline CLDN-5 & The absence of CLDN-5 may indicate damage to endothelial cells during sepsis & {$[31]$} \\
\hline \multirow[t]{3}{*}{ OCLN } & Increase related to sepsis severity and positive correlation with SOFA scores & {$[31,32]$} \\
\hline & Predictive of mortality & \\
\hline & $\begin{array}{l}\text { The absence of OCLN in the cerebral microvascular endothelium was related to more } \\
\text { severe disease and intense inflammatory response }\end{array}$ & \\
\hline \multirow[t]{3}{*}{ PAl-1 } & Sepsis severity prognosis & {$[33,34]$} \\
\hline & Predictor of mortality & \\
\hline & An increase may indicate DIC & \\
\hline \multirow[t]{2}{*}{ SICAM-1 } & Sepsis severity prognosis & {$[33,79]$} \\
\hline & Prognosis of 90-day mortality in patients with sepsis and septic shock in the ICU & \\
\hline \multirow[t]{4}{*}{ S100B } & It is associated with delirium in septic shock & {$[36,37,80]$} \\
\hline & Prognosis of severe organ dysfunction & \\
\hline & Shortest survival in 180 days & \\
\hline & Diagnosis of sepsis-associated encephalopathy & \\
\hline \multirow[t]{2}{*}{ E-selectin } & Sepsis severity prognosis & [33] \\
\hline & Predicts mortality & \\
\hline
\end{tabular}


Table 1 (continued)

\begin{tabular}{|c|c|c|}
\hline Biomarker & Function & References \\
\hline & Increase related to SOFA and APACHE-II & \\
\hline \multirow[t]{2}{*}{ sFlt-1 } & Prognosis of sepsis severity and SOFA score, & {$[33]$} \\
\hline & The prognosis for morbidity and mortality & \\
\hline \multirow[t]{3}{*}{ sVCAM-1 } & Prognosis of sepsis severity and 28-day mortality & {$[33,79,81]$} \\
\hline & Prognosis of 90-day mortality in patients with severe sepsis and septic shock in the ICU & \\
\hline & Risk of septic shock & \\
\hline \multirow[t]{3}{*}{ ZO-1 } & Prognosis of sepsis severity and correlation with APACHE-II and SOFA scores & {$[31,32]$} \\
\hline & Predictor of mortality & \\
\hline & Diagnostic capability for MODS & \\
\hline \multicolumn{3}{|c|}{ Gut permeability markers } \\
\hline Citrulline & The decrease may indicate early acute bowel dysfunction & {$[82,83]$} \\
\hline \multirow[t]{2}{*}{ I-FABP } & Risk of septic shock & {$[40]$} \\
\hline & Indicates early intestinal damage in patients with sepsis and septic shock & \\
\hline Zonulin & Indicates intestinal permeability during sepsis and SIRS & [39] \\
\hline D-lactic acid & Indicates early intestinal damage in patients with sepsis and septic shock & {$[40]$} \\
\hline \multicolumn{3}{|l|}{ Non-coding RNAs } \\
\hline \multirow[t]{6}{*}{ Lnc-MALAT1 } & The distinction between septic and non-sepsis patients & {$[41,42]$} \\
\hline & Positive correlation with APACHE-\| & \\
\hline & Sepsis severity prognosis & \\
\hline & High risk of ARDS & \\
\hline & Predictive for high mortality & \\
\hline & The increase can distinguish ARDS from non-ARDS & \\
\hline \multirow[t]{2}{*}{ Inc-MEG3 } & The increase is predictive of sepsis & {$[43]$} \\
\hline & 28-day mortality risk & \\
\hline \multicolumn{3}{|l|}{ miRNA } \\
\hline \multirow[t]{4}{*}{ miR-125a, miR-125b } & Prognosis of more significant disease severity & {$[44,84,85]$} \\
\hline & Distinguishes patients with sepsis from patients without sepsis & \\
\hline & miR-125b: increased risk of mortality in patients with sepsis & \\
\hline & miR-125a: risk of sepsis and increased mortality & \\
\hline \multicolumn{3}{|c|}{ Membrane receptors, cell proteins, and metabolites } \\
\hline \multirow[t]{3}{*}{ CD64 } & Prognosis of disease severity & {$[46]$} \\
\hline & 28-day mortality predictor & \\
\hline & Early diagnosis of infection & \\
\hline \multirow[t]{2}{*}{ CD68 } & Prognosis of disease severity & {$[86]$} \\
\hline & Microglial activation & \\
\hline NFL & Indicates risk and severity of sepsis-associated encephalopathy & {$[87]$} \\
\hline $\mathrm{NFH}$ & Indicates risk and severity of sepsis-associated encephalopathy & {$[87]$} \\
\hline \multirow[t]{4}{*}{ NSE } & Diagnosis of sepsis-associated encephalopathy & {$[80,88]$} \\
\hline & 30-day mortality risk & \\
\hline & Risk of delirium & \\
\hline & Neuronal injury marker in sepsis & \\
\hline \multirow[t]{2}{*}{ Presepsin } & Initial diagnosis and sepsis risk stratification & {$[48]$} \\
\hline & Potential marker to distinguish Gram (+) and Gram (-) bacterial infection & \\
\hline \multirow[t]{3}{*}{ TREM-1 } & Sepsis indicator & [89-91] \\
\hline & An early distinction between sepsis and SIRS & \\
\hline & Predictive of septic shock & \\
\hline \multicolumn{3}{|c|}{ Peptide precursor of the hormone and hormone } \\
\hline \multirow[t]{2}{*}{ MR-proADM } & Discrimination of survivors and non-survivors & [92] \\
\hline & Organ dysfunction marker & \\
\hline
\end{tabular}


Table 1 (continued)

\begin{tabular}{|c|c|c|}
\hline Biomarker & Function & References \\
\hline \multirow[t]{2}{*}{ PCT } & Diagnosis of sepsis & {$[66,90]$} \\
\hline & Predicts Bacterial Infection & \\
\hline \multirow[t]{2}{*}{ NT-proBNP } & $\begin{array}{l}\text { In the acute phase of sepsis it indicates a risk of long-term impairment of physical func- } \\
\text { tion and muscle strength }\end{array}$ & [55] \\
\hline & Predict mortality risk & [52] \\
\hline \multicolumn{3}{|c|}{ Neutrophil, cells, and related biomarkers } \\
\hline \multirow[t]{2}{*}{ Lactate } & Predictive of mortality & [93] \\
\hline & Risk stratification of patients with suspected infection & \\
\hline \multirow[t]{5}{*}{ MPO } & Increase in patients with DIC & {$[94,95]$} \\
\hline & Indicates organ dysfunction & \\
\hline & Mortality predictor at 28 and 90 days & \\
\hline & Risk of septic shock & \\
\hline & NET generation & \\
\hline \multirow[t]{3}{*}{ Resistin } & Sepsis indicator & {$[96,97]$} \\
\hline & Risk of septic shock & \\
\hline & 28-day mortality predictor & \\
\hline \multicolumn{3}{|c|}{ Soluble receptors } \\
\hline \multirow[t]{3}{*}{ sPD-L1 } & Prognosis of disease severity & {$[4,68]$} \\
\hline & 28-day mortality predictor & \\
\hline & Indicates immunosuppression & \\
\hline \multirow[t]{2}{*}{ SUPAR } & Predictive mortality at 7 and 30 days & [66] \\
\hline & Risk of patients with suspected infection & \\
\hline \multirow[t]{3}{*}{ sTNFR-1 } & Prognosis of 28-day mortality & {$[81,98]$} \\
\hline & Risk of septic shock & \\
\hline & Risk of delirium & \\
\hline \multicolumn{3}{|l|}{ Lipoproteins } \\
\hline \multirow[t]{2}{*}{ LDL-C } & Protective effect against sepsis & [99] \\
\hline & The decrease can cause a risk of sepsis and admission to the ICU & \\
\hline \multirow[t]{2}{*}{$\mathrm{HDL}$} & Low levels: mortality prognosis and adverse clinical outcomes & {$[100,101]$} \\
\hline & Predictive for MODS & \\
\hline T-chol & The decrease has a worse prognosis in sepsis & [102] \\
\hline \multicolumn{3}{|c|}{ 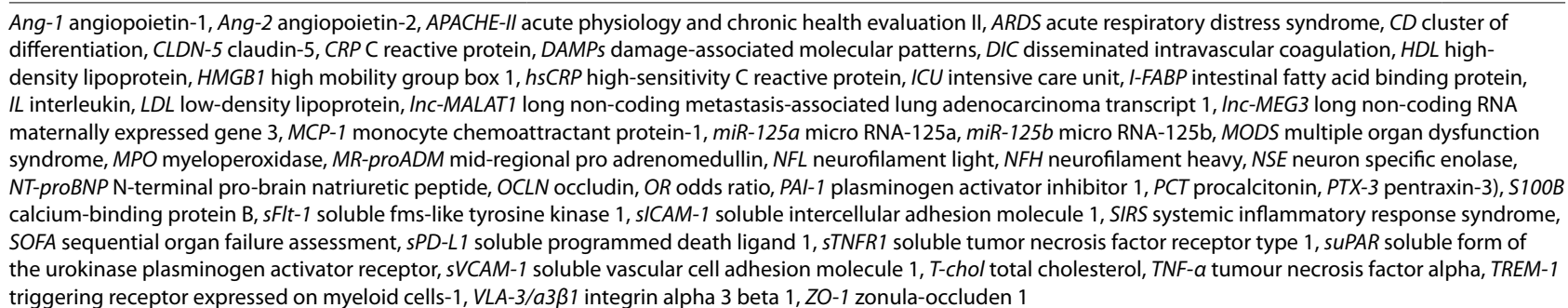 } \\
\hline
\end{tabular}

mortality discrimination compared to clinical variables alone [64]. In a multicenter prospective cohort study, soluble tumor necrosis factor receptor type 1 (sTNFR1) levels $>8861 \mathrm{pg} / \mathrm{ml}$ predicted 30-day mortality [65].

Patients with sepsis or septic shock displayed higher levels of the soluble form of the urokinase plasminogen activator receptor (suPAR), PCT, and lactate on days 1, 2, 4 , and 7 of admission, with lactate and suPAR being the best risk stratifies for suspected infection [66]. Levels of
suPAR and PCT levels were higher in sepsis patients than in a SIRS group with AUROC values of 0.89 and 0.82 , respectively [67]. Serum sPD-L1 levels were increased in non-survivors compared with survivors with similar prognostic accuracy for 28-day mortality as APACHEII and SOFA scores [68]. See Tables 1 and 2 for further information, as well as Fig. 1. 
Table 2 Biomarkers for sepsis, septic shock, and sepsis-associated encephalopathy

\begin{tabular}{|c|c|c|c|c|c|c|c|c|c|}
\hline Biomarker & Sample & Demographic & $\begin{array}{l}\text { Specificity } \\
(\%)\end{array}$ & $\begin{array}{l}\text { Sensitivity } \\
(\%)\end{array}$ & Cut-off & $R^{2}$ & AUC & Clinical relevance & References \\
\hline \multicolumn{10}{|c|}{ Acute-phase proteins } \\
\hline \multirow[t]{32}{*}{ CRP, hsCRP } & \multirow[t]{3}{*}{ Plasma and serum } & Sepsis $=483$ & - & - & $15-20 \mathrm{mg} / \mathrm{dl}$ & - & - & $\begin{array}{l}\uparrow \text { hsCRP hyperinflamma- } \\
\text { tory phenotype }\end{array}$ & \multirow[t]{3}{*}{ [22] } \\
\hline & & Mean age mean $=60.5$ & - & - & - & - & - & $\begin{array}{l}\uparrow \text { hsCRP day } 1 \text { to } 2 \text {, } \\
95.8 \%\end{array}$ & \\
\hline & & đ $54.9 \%$ & - & - & - & - & - & $\begin{array}{l}\uparrow \text { hsCRP, } 23 \text { patients } \\
(25.8 \%) \text { at } 3,26 \text { patients } \\
(30.2 \%) \text { at } 6 \text {, and } 23 \\
\text { patients }(25.6 \%) \text { at } \\
12 \text { months }\end{array}$ & \\
\hline & \multirow[t]{3}{*}{ Plasma } & Sepsis $=43$ & \multirow[t]{3}{*}{-} & \multirow[t]{3}{*}{ - } & \multirow[t]{3}{*}{-} & \multirow[t]{3}{*}{-} & \multirow{3}{*}{$\begin{array}{l}0.51,0.56 \text {, } \\
\text { and } 0.48\end{array}$} & \multirow{3}{*}{$\begin{array}{l}\text { CRP, day } 1,3 \text {, and } 8 \text { to } \\
\text { predict } 30 \text {-day mortality } \\
p=0.836, p=0.059 \text {, and } \\
p=0.819 \text {, respectively }\end{array}$} & \multirow[t]{3}{*}{ [74] } \\
\hline & & Septic shock $n=93$ & & & & & & & \\
\hline & & Age $=26$ to 88 & & & & & & & \\
\hline & \multirow[t]{4}{*}{ Plasma } & Sepsis $=17$ & \multirow[t]{4}{*}{$61.54 \%$} & \multirow[t]{4}{*}{$52.17 \%$} & \multirow[t]{4}{*}{$9 \mathrm{mg} / \mathrm{dl}$} & \multirow[t]{4}{*}{-} & \multirow[t]{4}{*}{0.684} & \multirow{4}{*}{$\begin{array}{l}\uparrow \text { hsCRP sepsis versus } \\
\text { control group, } p=0.008\end{array}$} & \multirow[t]{4}{*}{ [103] } \\
\hline & & Control $=19$ & & & & & & & \\
\hline & & Age $=52.18$ & & & & & & & \\
\hline & & \$ $63 \%$ & & & & & & & \\
\hline & \multirow[t]{4}{*}{ Serum } & Sepsis $=38$ & \multirow[t]{4}{*}{$100 \%$} & \multirow[t]{4}{*}{$88.40 \%$} & \multirow[t]{4}{*}{$8.02 \mathrm{mg} / \mathrm{l}$} & \multirow[t]{4}{*}{-} & \multirow[t]{4}{*}{0.98} & \multirow{4}{*}{$\begin{array}{l}\uparrow C R P \text { in septic patients } \\
\text { compared to control } \\
\text { group, } p=0.001\end{array}$} & [104] \\
\hline & & $\begin{array}{l}\text { Septic shock=31 Con- } \\
\text { trol }=40\end{array}$ & & & & & & & \\
\hline & & Age $=37$ to 95 & & & & & & & \\
\hline & & ○ $57.89 \%$ & & & & & & & \\
\hline & Serum & Sepsis $=27$ & $75.00 \%$ & $78.00 \%$ & $7.4 \mathrm{mg} / \mathrm{dl}$ & - & 0.825 & $\begin{array}{l}\uparrow \mathrm{hsCRP} \text { sepsis versus } \\
\text { control group, } p=0.001\end{array}$ & {$[105]$} \\
\hline & & Septic shock $=23$ & - & - & - & - & 0.751 & $\begin{array}{l}\uparrow \text { hsCRP septic shock } \\
\text { versus sepsis, } p=0.002\end{array}$ & \\
\hline & & Control $=20$ & - & - & - & 0.686 & - & $\begin{array}{l}\uparrow \text { hs-CRP level versus } \\
\text { SOFA, } p<0.001\end{array}$ & \\
\hline & & Age $=85$ & & & & & & & \\
\hline & & $\$ 57.89 \%$ & & & & & & & \\
\hline & Blood & Sepsis $=33$ & $90.70 \%$ & $98.60 \%$ & $407 \mathrm{pg} / \mathrm{ml}$ & - & 0.859 & $\uparrow$ CRP in sepsis patients & {$[48]$} \\
\hline & & Severe sepsis $=24$ & & & & & & compared in SIRS group, & \\
\hline & & Septic shock $=15$ & & & & & & & \\
\hline & & $\mathrm{SIRS}=23$ & & & & & & & \\
\hline & & Normal $=20$ & & & & & & & \\
\hline & & Mean age $=62.1$ & & & & & & & \\
\hline & Serum & Sepsis $=119$ & - & - & - & - & - & $\begin{array}{l}\uparrow C R P \text { and SOFA score } \\
\text { in the sepsis group } \\
\text { compared to the control } \\
\text { group, } p<0.05\end{array}$ & {$[46]$} \\
\hline & & Septic shock $=32$ & & & & & & $\begin{array}{l}\leftrightarrow \text { Septic shock group } \\
\text { when compared with } \\
\text { sepsis group, } p=0.086\end{array}$ & \\
\hline & & Control $=20$ & - & - & - & - & - & $\begin{array}{l}\leftrightarrow \text { Diagnosing positive } \\
\text { infection culture in } \\
\text { patients with sepsis, } \\
p=0.071\end{array}$ & \\
\hline & & & - & - & - & - & 0.609 & & \\
\hline & Serum & Severe sepsis $=34$ & - & - & - & - & - & $\leftrightarrow$ CRP did not differenti- & [89] \\
\hline & & Septic shock $=53$ & & & & & & $\begin{array}{l}\text { ate septic shock and } \\
\text { severe sepsis }\end{array}$ & \\
\hline & & $\mathrm{Age}=2 \mathrm{mo}$ to 16 years & & & & & & & \\
\hline
\end{tabular}


Table 2 (continued)

\begin{tabular}{|c|c|c|c|c|c|c|c|c|c|}
\hline Biomarker & Sample & Demographic & $\begin{array}{l}\text { Specificity } \\
(\%)\end{array}$ & $\begin{array}{l}\text { Sensitivity } \\
(\%)\end{array}$ & Cut-off & $R^{2}$ & AUC & Clinical relevance & References \\
\hline $\begin{array}{l}\text { Comple- } \\
\text { ment }\end{array}$ & Plasma & Sepsis $=20$ & - & - & - & - & - & $\begin{array}{l}\uparrow \text { Sepsis (C4d 3.5-fold; } \\
\text { Factor Bb 6.1-fold; C3 } \\
0.8 \text {-fold; C3a 11.6-fold; } \\
\text { and C5a 1.8-fold) versus } \\
\text { control }\end{array}$ & [25] \\
\hline \multirow[t]{3}{*}{ Proteins } & & Control $=10$ & & & & & & $\uparrow$ C5a $\downarrow$ SOFA & \\
\hline & & Age $=85$ & - & - & - & 0.18 & - & & \\
\hline & & $\$ 57.89 \%$ & & & & & - & & \\
\hline \multirow[t]{9}{*}{ PTX-3 } & Plasma & Sepsis $=73$ & - & - & - & 0.36 & - & $\begin{array}{l}\uparrow P T X-3 \text { versus APACHE- } \\
\| 1, \text { and SOFA, } p=0.0001\end{array}$ & [72] \\
\hline & & Control $=77$ & - & - & $31.4 \mathrm{ng} / \mathrm{ml}$ & - & - & $\begin{array}{l}\text { Sepsis versus SIRS, } \\
p>0.05\end{array}$ & \\
\hline & & Septic Shock $=140$ & - & - & - & - & - & $\begin{array}{l}\text { Sepsis versus septic } \\
\text { shock, } p=0.0001\end{array}$ & \\
\hline & & Age $=26$ to 88 & - & - & - & - & - & $\begin{array}{l}\uparrow \text { Sepsis/septic shock } \\
\text { versus control, } p \leq 0.001\end{array}$ & \\
\hline & & $\$ 57.89 \%$ & - & - & - & - & $\begin{array}{l}0.82 \text { and } \\
0.73\end{array}$ & $\begin{array}{l}\text { Sepsis and septic shock } \\
\text { discrimination on day } 1\end{array}$ & \\
\hline & Plasma & Sepsis $=17$ & - & - & - & - & - & $\begin{array}{l}\uparrow \text { PTX-3 sepsis, septic } \\
\text { shock, and post-surgery } \\
\text { infection versus control } \\
\text { group, } p<0.05\end{array}$ & [73] \\
\hline & & Septic shock $=26$ & & & & & & $\begin{array}{l}\uparrow P T X-3 \text { sepsis shock } \\
\text { versus sepsis, } p<0.0001\end{array}$ & \\
\hline & & Post-surg. Inf. $=33$ & - & - & - & - & 0.798 & & \\
\hline & & Control $=25$ & & & & & & & \\
\hline \multicolumn{10}{|c|}{ Cytokines and chemokines } \\
\hline \multirow[t]{5}{*}{ IL-10 } & Plasma & $\begin{array}{l}\text { Sepsis }=208 \text { Con- } \\
\text { trol }=210\end{array}$ & - & - & - & - & -0.161 & $\begin{array}{l}\uparrow \text { miR-126 correlated } \\
\text { negatively with IL-10, } \\
p=0.020\end{array}$ & {$[106]$} \\
\hline & Plasma & Sepsis $=309$ & - & - & - & - & -0.166 & 个 IncRNA ITSN1-2 cor- & [107] \\
\hline & & Mean age $=57,3 \pm 9,7$ & & & & & & 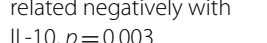 & \\
\hline & & Control $=330$ & & & & & & & \\
\hline & & Mean age $=55,8 \pm 9,7$ & & & & & & & \\
\hline \multirow[t]{7}{*}{ MCP-1 } & Plasma & Sepsis $=43$ & - & - & - & - & $0.64,0.51$ & MCP-1, day 1,3 , and 8 to & [74] \\
\hline & & Septic shock $n=93$ & & & & & and 0.51 & predict 30-day mortality, & \\
\hline & & Age $=26$ to 88 & & & & & & $p=0.948$, respectively & \\
\hline & Plasma & Sepsis $=17$ & - & - & - & - & - & $\begin{array}{l}\uparrow M C P-1 \text { sepsis, septic } \\
\text { shock and post-surgery } \\
\text { infection versus control } \\
\text { group, } p<0.05\end{array}$ & [73] \\
\hline & & Septic shock $=26$ & & & & & & $\begin{array}{l}\uparrow M C P-1 \text { sepsis shock } \\
\text { versus sepsis, } p=0.0059\end{array}$ & \\
\hline & & Post-surg. Inf. $=33$ & - & - & - & - & 0.71 & & \\
\hline & & Control $=25$ & & & & & & & \\
\hline \multirow[t]{6}{*}{$\begin{array}{l}\text { TNF- } a, \text { IL-1 } \beta, \\
\text { IL-6 }\end{array}$} & Serum & Sepsis $=288$ & - & - & - & - & - & $\begin{array}{l}\uparrow \text { Sepsis TNF-a, IL-1 } \beta, I L-6 \text {, } \\
\text { and IL-8 compared to the } \\
\text { control group, } p<0.001\end{array}$ & [70] \\
\hline & & Mean age $=58.2 \pm 11.2$ & & & & & & $\begin{array}{l}\uparrow \text { TNF-a, IL-1 } 1 \beta, \text { LL-6, and } \\
\text { IL-8 were negatively } \\
\text { correlated with surviving } \\
\text { sepsis patients, } p<0.001\end{array}$ & \\
\hline & & Control $=290$ & - & - & - & - & -0.270 & & \\
\hline & & Mean age $=56.8 \pm 12.1$ & & & & & -0.310 & & \\
\hline & & & & & & & $\begin{array}{l}-0.254 \\
\text { and }\end{array}$ & & \\
\hline & & & & & & & -0.256 & & \\
\hline
\end{tabular}


Table 2 (continued)

\begin{tabular}{|c|c|c|c|c|c|c|c|c|c|}
\hline Biomarker & Sample & Demographic & $\begin{array}{l}\text { Specificity } \\
\text { (\%) }\end{array}$ & $\begin{array}{l}\text { Sensitivity } \\
\text { (\%) }\end{array}$ & Cut-off & $R^{2}$ & AUC & Clinical relevance & References \\
\hline & Plasma & $\begin{array}{l}\text { Sepsis }=483 \\
\text { Age mean }=60.5 \\
\text { o } 54.9 \%\end{array}$ & - & - & $10^{2} \mathrm{pg} / \mathrm{dl}$ & - & - & $\begin{array}{l}\uparrow I \mathrm{IL}-6,72 \text { patients } \\
(74.2 \%) \text { at } 3 \text { months, } 62 \\
(70.5 \%) \text { at } 6 \text { months, and } \\
59(66.3 \%) \text { at } 12 \text { months }\end{array}$ & [22] \\
\hline & Serum & $\begin{array}{l}\text { Sepsis }=43 \\
\text { Septic shock } n=93 \\
\text { Age }=26 \text { to } 88\end{array}$ & - & - & - & - & $\begin{array}{l}0.69,0.70 \\
\text { and } 0.68\end{array}$ & $\begin{array}{l}\uparrow I L-6 \text {, day } 1,3 \text {, and } 8 \text { to } \\
\text { predict } 30 \text {-day mortality, } \\
p=0.0001, p=0.0001 \text {, } \\
\text { and } p=0.012 \text {, respec- } \\
\text { tively }\end{array}$ & [74] \\
\hline & \multirow[t]{3}{*}{ Serum } & Sepsis $=39$ & \multirow[t]{3}{*}{-} & \multirow[t]{3}{*}{-} & $\begin{array}{l}12,704- \\
111,372\end{array}$ & \multirow[t]{3}{*}{-} & \multirow[t]{3}{*}{-} & \multirow[t]{3}{*}{$\begin{array}{l}\text { 个 IL-6 septic patients } \\
\text { with DIC, } p=0.01\end{array}$} & \multirow[t]{4}{*}{ [34] } \\
\hline & & Control $=15$ & & & \multirow[t]{2}{*}{$\mathrm{pg} / \mathrm{ml}$} & & & & \\
\hline & & Age $\geq 18$ years & & & & & & & \\
\hline \multicolumn{9}{|l|}{ DAMPs } & \\
\hline \multirow[t]{2}{*}{ Calprotectin } & \multirow[t]{2}{*}{ Plasma } & Sepsis $=77$ & $56 \%$ & $81 \%$ & $1.3 \mathrm{mg} / \mathrm{l}$ & - & - & $\begin{array}{l}\uparrow \text { Calprotectin, sepsis } \\
\text { versus trauma patients, } \\
p<0.001\end{array}$ & \multirow[t]{2}{*}{ [28] } \\
\hline & & Trauma $=32$ & - & - & - & - & - & $\begin{array}{l}\uparrow \text { Calprotectin at admis- } \\
\text { sion was } \uparrow \text { in non-survi- } \\
\text { vors than in survivors at } \\
\text { day } 30, p<0.01\end{array}$ & \\
\hline \multirow[t]{3}{*}{ HMGB-1 } & \multirow[t]{3}{*}{ Serum } & \multirow[t]{3}{*}{ Sepsis $=247$} & - & - & $3.6 \mathrm{ng} / \mathrm{ml}$ & - & - & $\begin{array}{l}\uparrow \text { HMGB-1 sepsis versus } \\
\text { control, } p<0.001\end{array}$ & \multirow[t]{3}{*}{ [75] } \\
\hline & & & - & - & - & - & $\begin{array}{l}0.51 \text { and } \\
0.53\end{array}$ & $\begin{array}{l}\text { HMGB-1, day } 0 \text { and } 3 \text {, } \\
\text { survivor = non-survivor }\end{array}$ & \\
\hline & & & - & - & - & - & - & $\begin{array}{l}\text { HMGB-1 does not } \\
\text { have predictive value } \\
\text { for organ failure and } \\
\text { outcome }\end{array}$ & \\
\hline Endothelial ce & Ills and BBB $n$ & & & & & & & & \\
\hline Syndecan-1 & Serum & Sepsis $=39$ & - & - & - & - & - & $\begin{array}{l}\uparrow \text { Syndecan-1 in sepsis } \\
\text { versus control, } p<0.0001\end{array}$ & [34] \\
\hline & & Control $=15$ & - & - & - & - & - & $\begin{array}{l}\uparrow \text { Syndecan-1 non- } \\
\text { survivors on days } 1,2 \text {, } \\
\text { and } 4\end{array}$ & \\
\hline & & Age & - & - & - & - & $\begin{array}{l}0.54 \text { and } \\
0.59\end{array}$ & $\begin{array}{l}\uparrow \text { Syndecan- } 1 \text { versus } \\
\text { DIC on day } 1 \text { and } \\
2, p=0.0004 \text { and } \\
p=0.0002 \text {, subsequently }\end{array}$ & \\
\hline & & Age $\geq 18$ years & - & - & $\begin{array}{l}\text { 189-1301 ng/ } \\
\mathrm{ml}\end{array}$ & - & - & $\begin{array}{l}\uparrow \text { Syndecan-1 in septic } \\
\text { patient with DIC, } p<0.01\end{array}$ & \\
\hline VLA-3 (a3ß1) & Neutrophil & $\mathrm{SIRS}=9$ & - & - & - & - & - & $\uparrow a 3 \beta 1$ (VLA-3, CD49c/ & [77] \\
\hline & & Sepsis $=15$ & & & & & & $\begin{array}{l}\text { CD29) on neutrophils of } \\
\text { septic patients, } p<0.05\end{array}$ & \\
\hline & & Control $=7$ & & & & & & & \\
\hline & & Sepsis $=6$ & - & - & - & - & - & $\uparrow \beta 1$ (CD29), on neutro- & [78] \\
\hline & & Control $=5$ & & & & & & $\begin{array}{l}\text { phils of sepsis patients, } \\
p<0.05\end{array}$ & \\
\hline Ang-1 & Serum & Severe sepsis $=48$ & - & - & - & - & - & $\begin{array}{l}\uparrow \text { Ang-1 severe sepsis } \\
\text { compared with shock } \\
\text { septic, } p<0.01\end{array}$ & [79] \\
\hline & & Septic shock $=54$ & & & & & & $\begin{array}{l}\downarrow \text { Ang-1/Tie-2 in non- } \\
\text { survivors, } p<0.001\end{array}$ & \\
\hline & & Age $\geq 18$ years & - & - & $\begin{array}{l}3.81-16.1 \mathrm{ng} / \\
\mathrm{ml}\end{array}$ & - & - & & \\
\hline
\end{tabular}


Table 2 (continued)

\begin{tabular}{|c|c|c|c|c|c|c|c|c|c|}
\hline Biomarker & Sample & Demographic & $\begin{array}{l}\text { Specificity } \\
\text { (\%) }\end{array}$ & $\begin{array}{l}\text { Sensitivity } \\
\text { (\%) }\end{array}$ & Cut-off & $R^{2}$ & AUC & Clinical relevance & References \\
\hline & Plasma & $\mathrm{SIRS}=943$ & - & - & - & - & - & $\begin{array}{l}\uparrow \text { Ang-1 was associated } \\
\text { with a reduced risk of } \\
\text { shock. OR: } 0.77\end{array}$ & [81] \\
\hline & & Sepsis $=330$ & & & & & & $\begin{array}{l}\uparrow \text { Ang-1 was higher in } \\
\text { survivor versus non- } \\
\text { survivor, } p<0.001\end{array}$ & \\
\hline & & Shock $=216$ & - & - & $5719 \mathrm{pg} / \mathrm{ml}$ & - & - & & \\
\hline & & Pneumonia = 169 & & & & & & & \\
\hline & & Others $=152$ & & & & & & & \\
\hline & & Age $=55.1 \pm 16.1$ & & & & & & & \\
\hline & & ot $63.9 \%$ & & & & & & & \\
\hline \multirow[t]{10}{*}{ Ang-2 } & Serum & Severe sepsis $=48$ & - & - & - & - & - & $\begin{array}{l}\uparrow \text { Ang-2 severe sepsis } \\
\text { compared with shock } \\
\text { septic, } p<0.02\end{array}$ & [79] \\
\hline & & Septic shock = 54 & & & & & & $\begin{array}{l}\downarrow \text { Ang-2/Ang-1 in non- } \\
\text { survivors, } p<0.001\end{array}$ & \\
\hline & & Age $\geq 18$ years & - & - & - & - & - & & \\
\hline & Plasma & $\mathrm{SIRS}=943$ & - & - & - & - & - & $\begin{array}{l}\uparrow \text { Ang- } 2 \text { was associated } \\
\text { with an increased risk of } \\
\text { shock, OR: } 1.63\end{array}$ & [81] \\
\hline & & Sepsis $=330$ & - & - & $42.063 \mathrm{pg} / \mathrm{ml}$ & - & - & $\begin{array}{l}\uparrow A n g-2 \text { non-survivor, } \\
p<0.001\end{array}$ & \\
\hline & & Shock $=216$ & & & & & & & \\
\hline & & Pneumonia $=169$ & & & & & & & \\
\hline & & Others $=152$ & & & & & & & \\
\hline & & Age $=55.1 \pm 16.1$ & & & & & & & \\
\hline & & ơ $63.9 \%$ & & & & & & & \\
\hline \multirow[t]{6}{*}{ CLDN-5 } & Serum & Sepsis $=11$ & - & - & - & - & - & $\begin{array}{l}\uparrow C L D N-5 \text { was not asso- } \\
\text { ciated with MODS and } \\
\text { the non-MODS group }\end{array}$ & [31] \\
\hline & & Severe sepsis $=18$ & - & - & - & - & $\begin{array}{l}0.157 \text { and } \\
0.087\end{array}$ & $\begin{array}{l}\uparrow C L D N-5 \text { was not } \\
\text { correlated with SOFA or } \\
\text { APACHE score, } p=0.270 \text {, } \\
p=0.542\end{array}$ & \\
\hline & & Septic shock $=22$ & - & - & - & - & - & Did not predict mortality & \\
\hline & Serum & Sepsis $=11$ & - & - & - & - & - & CLDN-5 was absent from & {$[32]$} \\
\hline & & Severe sepsis $=18$ & & & & & & the endothelium & \\
\hline & & Septic shock $=22$ & & & & & & & \\
\hline \multirow[t]{4}{*}{ OCLN } & Serum & Sepsis $=11$ & - & - & - & - & - & $\begin{array}{l}\uparrow \text { OCLN in severe sepsis } \\
\text { and septic shock than in } \\
\text { sepsis, } p<0.05\end{array}$ & [31] \\
\hline & & Severe sepsis $=18$ & - & - & - & - & - & $\begin{array}{l}\uparrow \text { OCLN in non-survivors } \\
\text { compared with survivors, } \\
p<0.01\end{array}$ & \\
\hline & & Septic shock $=22$ & - & - & - & - & 0.337 & $\begin{array}{l}\uparrow \text { OCLN positively } \\
\text { correlated with SOFA, } \\
p<0.016\end{array}$ & \\
\hline & & & - & - & - & - & 0.224 & $\begin{array}{l}\leftrightarrow \text { OCLN levels were } \\
\text { not correlated with the } \\
\text { APACHE-II, } p<0.085\end{array}$ & \\
\hline
\end{tabular}


Table 2 (continued)

\begin{tabular}{|c|c|c|c|c|c|c|c|c|c|}
\hline Biomarker & Sample & Demographic & $\begin{array}{l}\text { Specificity } \\
\text { (\%) }\end{array}$ & $\begin{array}{l}\text { Sensitivity } \\
\text { (\%) }\end{array}$ & Cut-off & $R^{2}$ & AUC & Clinical relevance & References \\
\hline & $\begin{array}{l}\text { Brain tissue } \\
\text { autopsies }\end{array}$ & Sepsis $=47$ & - & - & - & - & - & $\begin{array}{l}\downarrow \text { OCLN, cerebellar } \\
\text { endothelium damage, } \uparrow \\
\mathrm{CRP} \geq 100 \mathrm{mg} / \mathrm{l}\end{array}$ & {$[32]$} \\
\hline & & & - & - & - & - & - & $\begin{array}{l}38 \% \text { of patients }(18 / 47) \\
\text { had no expression of } \\
\text { OCLN in the BMVECs }\end{array}$ & \\
\hline & & & - & - & - & - & - & $\begin{array}{l}34 \% \text { of patients }(16 / 47) \\
\text { had MOFs }\end{array}$ & \\
\hline & & & - & - & - & - & - & $\begin{array}{l}74.5 \% \text { of patients ( } 35 / 47) \\
\text { had septic shock }\end{array}$ & \\
\hline & & & - & - & - & - & - & $\begin{array}{l}\text { The deceased with BBB } \\
\text { damage had SOFA scores } \\
\text { six versus } 14, p=0.04\end{array}$ & \\
\hline \multirow[t]{7}{*}{ PAl-1 } & Plasma & Sepsis $=63$ & - & - & - & - & 0.85 & $\begin{array}{l}\uparrow \text { PAl-1 to predict mortal- } \\
\text { ity, } p<0.05\end{array}$ & [33] \\
\hline & & Severe sepsis $=61$ & - & - & - & 0.45 & - & $\begin{array}{l}\uparrow \text { PAl-1 correlated with } \\
\text { the SOFA score at } 24 \mathrm{~h}, \\
p<0.0001\end{array}$ & \\
\hline & & Septic shock $=42$ & - & - & - & 0.58 & - & $\begin{array}{l}\uparrow \text { PAI-1 correlation } \\
\text { with APACHE-II score, } \\
p<0.0001\end{array}$ & \\
\hline & & Age $=60 \pm 17$ & - & - & - & - & - & $\begin{array}{l}\uparrow \text { Severe sepsis } \uparrow \text { sFlt- } 1, \\
p<0.0001\end{array}$ & \\
\hline & Serum & Sepsis $=39$ & - & - & $15.5-49.9$ & - & - & $\uparrow$ PAI- 1 in patients with & [34] \\
\hline & & Control $=15$ & & & $\mathrm{ng} / \mathrm{ml}$ & & & $\mathrm{DIC}, p=0.016$ & \\
\hline & & Age $\geq 18$ years & & & & & & & \\
\hline \multirow[t]{7}{*}{ sICAM-1 } & Plasma & Sepsis $=63$ & - & - & - & - & - & $\begin{array}{l}\uparrow \text { Severe sepsis } \uparrow \text { sICAM- } \\
1, p<0.001\end{array}$ & [33] \\
\hline & & Severe sepsis $=61$ & - & - & - & 0.15 & - & $\begin{array}{l}\uparrow \text { sICAM-1 correlated } \\
\text { with SOFA score at } 24 \mathrm{~h}, \\
p<0.03\end{array}$ & \\
\hline & & Septic shock $=42$ & & & & & - & $\begin{array}{l}\uparrow \text { sICAM-1 correlate with } \\
\text { APACHE-II score, } p<0.05\end{array}$ & \\
\hline & & Age $=60 \pm 17$ & - & - & - & 0.17 & - & & \\
\hline & Serum & Severe sepsis $=48$ & - & - & $\begin{array}{l}1.297- \\
1787 \mathrm{ng} / \mathrm{ml}\end{array}$ & - & - & $\begin{array}{l}\uparrow \text { sICAM-1 in non- } \\
\text { survivors, } p<0.001\end{array}$ & [79] \\
\hline & & Septic shock $=54$ & - & - & - & - & - & $\begin{array}{l}\uparrow \text { sICAM-1, predictor } \\
\text { of } 90 \text { day-mortality, } \\
p<0.0001\end{array}$ & \\
\hline & & Age $\geq 18$ years & - & - & - & - & - & $\begin{array}{l}\uparrow \text { sICAM-1, septic shock } \\
\text { compared to severe } \\
\text { sepsis } p<0.01\end{array}$ & \\
\hline \multirow[t]{3}{*}{ S100B } & Serum & Septic shock $=22$ & - & - & $>0.15 \mu \mathrm{g} / \mathrm{l}$ & - & - & $\begin{array}{l}\uparrow \text { Delirium was present } \\
\text { in } 10 / 22 \text { of the patients } \\
(45.5 \%)\end{array}$ & [37] \\
\hline & & & - & - & - & - & - & $\begin{array}{l}\text { OR: } 18.0, \text { for risk of } \\
\text { developing delirium } \\
\text { S-100ß }>0.15 \mu \mathrm{g} / \mathrm{l}\end{array}$ & \\
\hline & & & - & - & - & - & 0.489 & $\begin{array}{l}\uparrow S 100 \beta \text { correlate } \\
\text { positively with and IL-6 } \\
p=0.021\end{array}$ & \\
\hline
\end{tabular}


Table 2 (continued)

\begin{tabular}{|c|c|c|c|c|c|c|c|c|c|}
\hline Biomarker & Sample & Demographic & $\begin{array}{l}\text { Specificity } \\
\text { (\%) }\end{array}$ & $\begin{array}{l}\text { Sensitivity } \\
\text { (\%) }\end{array}$ & Cut-off & $R^{2}$ & AUC & Clinical relevance & References \\
\hline & \multirow[t]{6}{*}{ Serum } & Sepsis $=104$ & $80.0 \%$ and & 66.1 and & 0.226 and & - & - & $\begin{array}{l}\uparrow S 100 \mathrm{~B} \text { cut-of value for } \\
\text { day } 1 \text { and } 3\end{array}$ & {$[36]$} \\
\hline & & $\begin{array}{l}\text { Sepsis-associated } \\
\text { encephalopathy }=59\end{array}$ & $84.44 \%$ & $69.49 \%$ & $0.144 \mu \mathrm{g} / \mathrm{l}$ & - & - & $\begin{array}{l}\uparrow S 100 B \text { in sepsis-asso- } \\
\text { ciated encephalopathy } \\
\text { day } 1 \text { to day } 3 \text { compared } \\
\text { with non- sepsis-asso- } \\
\text { ciated encephalopathy, } \\
p<0.001\end{array}$ & \\
\hline & & \multirow[t]{4}{*}{$\begin{array}{l}\text { non- sepsis-associated } \\
\text { encephalopathy }=45\end{array}$} & - & - & - & - & $\begin{array}{l}0.728 \text { and } \\
0.819\end{array}$ & $\begin{array}{l}\uparrow S 100 B \text { on days } 1 \text { and } 3 \\
\text { to predict sepsis-associ- } \\
\text { ated encephalopathy }\end{array}$ & \\
\hline & & & - & - & - & - & 0.61 & $\begin{array}{l}\uparrow S 100 B \text { on day } 1 \text { to pre- } \\
\text { dict } 180 \text { day-mortality }\end{array}$ & \\
\hline & & & $84.44 \%$ & $69.49 \%$ & $0.529 \mu \mathrm{g} / \mathrm{l}$ & - & 0.731 & $\begin{array}{l}\uparrow \mathrm{S} 100 \mathrm{~B} \text { on day } 3 \text { to pre- } \\
\text { dict } 180 \text { day-mortality }\end{array}$ & \\
\hline & & & $93.33 \%$ & $50.00 \%$ & $0.266 \mu \mathrm{g} / \mathrm{l}$ & & & & \\
\hline & \multirow[t]{2}{*}{ Serum } & Sepsis $=21$ & - & - & - & - & $\begin{array}{l}0.082 \\
0.082\end{array}$ & $\begin{array}{l}\uparrow S 100 B \text { did not correlate } \\
\text { with GCS, EEG pattern, or }\end{array}$ & {$[108]$} \\
\hline & & Age $=68.7$ & & & & & and 0.124 & SOFA scores & \\
\hline \multirow[t]{4}{*}{ E-selectin } & \multirow[t]{4}{*}{ Plasma } & Sepsis $=63$ & - & - & - & - & 0.77 & $\uparrow$ Predict mortality & [33] \\
\hline & & Severe sepsis $=61$ & - & - & - & - & - & $\begin{array}{l}\uparrow \text { Severe sepsis } \uparrow \text { sE- } \\
\text { selectin, } p<0.001\end{array}$ & \\
\hline & & Septic shock $=42$ & - & - & - & 0.27 & - & $\begin{array}{l}\uparrow s \text { sE-selectin correlated } \\
\text { with SOFA score at } 24 \mathrm{~h} \text {, } \\
p<0.0001\end{array}$ & \\
\hline & & Age $=60 \pm 17$ & - & - & - & 0.31 & - & $\begin{array}{l}\uparrow s \text { sE-selectin correlated } \\
\text { with APACHE-II score, } \\
p<0.0001\end{array}$ & \\
\hline \multirow[t]{5}{*}{ sFlt-1 } & \multirow[t]{5}{*}{ Plasma } & Sepsis $=63$ & - & - & - & - & 0.85 & $\begin{array}{l}\uparrow s \text { sFlt-1 to predict mor- } \\
\text { tality, } p<0.05\end{array}$ & [33] \\
\hline & & Severe sepsis $=61$ & - & - & - & 0.36 & - & $\begin{array}{l}\uparrow s \text { sflt-1 associated with } \\
\text { organ dysfunction }\end{array}$ & \\
\hline & & Septic shock $=42$ & - & - & - & 0.63 & - & $\begin{array}{l}\uparrow \text { sFlt-1 correlation with } \\
\uparrow \mathrm{IL}-6, p<0.0001\end{array}$ & \\
\hline & & Age $=60 \pm 17$ & - & - & - & 0.6 & - & $\begin{array}{l}\uparrow \text { sFlt-1 correlated with } \\
\text { SOFA score at } 24 \mathrm{~h}, \\
p<0.0001\end{array}$ & \\
\hline & & & - & - & - & 0.64 & - & $\begin{array}{l}\uparrow \text { sFlt-1 correlated } \\
\text { with APACHE-II score, } \\
p<0.0001\end{array}$ & \\
\hline \multirow[t]{7}{*}{ sVCAM-1 } & \multirow[t]{4}{*}{ Plasma } & Sepsis $=63$ & - & - & - & - & 0.78 & $\uparrow$ Predict mortality & {$[33]$} \\
\hline & & Severe sepsis $=61$ & - & - & - & - & - & $\begin{array}{l}\uparrow \text { Severe sepsis } \uparrow \\
\text { sVCAM-1, } p<0.002\end{array}$ & \\
\hline & & Septic shock $=42$ & - & - & - & 0.45 & - & $\begin{array}{l}\uparrow \mathrm{sE} \text {-selectin correlated } \\
\text { with SOFA at } 24 \mathrm{~h}, \\
p<0.0001\end{array}$ & \\
\hline & & Age $=60 \pm 17$ & - & - & - & 0.38 & - & $\begin{array}{l}\uparrow \text { sVCAM-1 correlated } \\
\text { with APACHE-II s, } \\
p<0.0001\end{array}$ & \\
\hline & \multirow[t]{3}{*}{ Serum } & Severe sepsis $=48$ & - & - & $369-467 \mu g / l$ & - & - & $\begin{array}{l}\uparrow \text { sVCAM in non-survi- } \\
\text { vors, } p<0.001\end{array}$ & [79] \\
\hline & & Septic shock $=54$ & - & - & - & - & - & $\begin{array}{l}\uparrow \text { sVCAM, predictor } \\
\text { of } 90 \text { day-mortality, } \\
p<0.0001\end{array}$ & \\
\hline & & Age $\geq 18$ years & - & - & - & - & - & $\begin{array}{l}\uparrow \text { sVCAM, septic shock } \\
\text { compared to severe } \\
\text { sepsis, } p<0.01\end{array}$ & \\
\hline
\end{tabular}


Table 2 (continued)

\begin{tabular}{|c|c|c|c|c|c|c|c|c|c|}
\hline Biomarker & Sample & Demographic & $\begin{array}{l}\text { Specificity } \\
\text { (\%) }\end{array}$ & $\begin{array}{l}\text { Sensitivity } \\
\text { (\%) }\end{array}$ & Cut-off & $R^{2}$ & AUC & Clinical relevance & References \\
\hline & Plasma & $\mathrm{SIRS}=943$ & - & - & - & - & - & $\begin{array}{l}\uparrow s-V C A M \text { was associated } \\
\text { with an increased risk of } \\
\text { shock. OR: } 1.63\end{array}$ & [81] \\
\hline & & Sepsis $=330$ & & & & & & $\begin{array}{l}\uparrow \text { sVCAM-1 non-survivor, } \\
p<0.001\end{array}$ & \\
\hline & & Shock $=216$ & - & - & $819 \mathrm{pg} / \mathrm{ml}$ & - & - & & \\
\hline & & Pneumonia $=169$ & & & & & & & \\
\hline & & Others $=152$ & & & & & & & \\
\hline & & Age $=55.1 \pm 16.1$ & & & & & & & \\
\hline & & o $63.9 \%$ & & & & & & & \\
\hline \multirow[t]{5}{*}{$\mathrm{ZO}-1$} & Serum & Sepsis $=11$ & - & - & - & - & - & $\begin{array}{l}\uparrow \text { ZO- } 1 \text { in severe sepsis } \\
\text { and septic shock com- } \\
\text { pared to sepsis, } p<0.05\end{array}$ & [31] \\
\hline & & Severe sepsis $=18$ & - & - & - & - & - & $\begin{array}{l}\uparrow \text { ZO- } 1 \text { in non-survivors } \\
\text { compared with survivors, } \\
p<0.01\end{array}$ & \\
\hline & & Septic shock $=22$ & - & - & - & - & - & $\uparrow Z O-1$ in MODs group & \\
\hline & & & - & - & - & - & $\begin{array}{l}0.502 \text { and } \\
0.380\end{array}$ & $\begin{array}{l}\uparrow \text { ZO- } 1 \text { was positively } \\
\text { correlated with SOFA } \\
\text { and APACHE-II scores, } \\
p<0.001 \text { and } p<0.006\end{array}$ & \\
\hline & $\begin{array}{l}\text { Brain tissue } \\
\text { autopsies }\end{array}$ & Sepsis $=47$ & - & - & - & - & - & $\begin{array}{l}\text { ZO- } 1 \text { is absent from } \\
\text { the endothelial cells } \\
\text { in the cerebrum and } \\
\text { endothelium }\end{array}$ & [32] \\
\hline \multicolumn{10}{|c|}{ Gut permeability markers } \\
\hline \multirow[t]{8}{*}{ Citrulline } & Plasma & Septic shock = 16 & - & - & - & - & - & $\begin{array}{l}\text { Citrulline was positively } \\
\text { correlated with plasma } \\
\text { arginine }\left(r^{2}=0.85\right) \text { and } \\
\text { glutamine }\left(r^{2}=0.90\right) \\
\text { concentrations in both } \\
\text { groups, and significantly } \\
\text { inversely correlated with } \\
\text { CRP }\left(r^{2}=0.10\right)\end{array}$ & {$[109]$} \\
\hline & & (Survivors $=8$ & & & & & & $\begin{array}{l}\downarrow \text { Citrulline in patients } \\
\text { with digestive bacterial } \\
\text { translocation }\end{array}$ & \\
\hline & & Age $=60 \pm 16.5$ & & & & & & & \\
\hline & & Non-survivor $=8$ & & & & & & & \\
\hline & & Age $=62.9 \pm 18.5$ & - & - & - & - & - & & \\
\hline & Plasma & Sepsis $/$ ARDS $=44$ & - & - & - & - & - & $\downarrow$ Citrulline in all patients & {$[83]$} \\
\hline & & Sepsis/NO ARDS = 91 & - & - & 6 and $10.1 \mathrm{uM}$ & - & - & $\begin{array}{l}\downarrow \text { ARDS compared to } \\
\text { the no ARDS group, } \\
p=0.002\end{array}$ & \\
\hline & & Age $=55 \pm 16$ & - & - & - & - & - & $\begin{array}{l}\text { Citrulline levels were } \\
\text { associated with ARDS }\end{array}$ & \\
\hline \multirow[t]{3}{*}{ I-FABP } & Serum & Sepsis $=30$ & - & - & $\begin{array}{l}27.46 \text { and } \\
36.95 \mu \mathrm{g} / \mathrm{l}\end{array}$ & - & - & $\begin{array}{l}\uparrow \text { I-FABP sepsis and sep- } \\
\text { tic shock group, } p<0.01\end{array}$ & {$[40]$} \\
\hline & & Septic shock $=30$ & & & - & & & $\begin{array}{l}\uparrow \text { I-FABP no difference } \\
\text { between survivors and } \\
\text { non-survivors }\end{array}$ & \\
\hline & & Control $=20$ & - & - & & - & - & & \\
\hline
\end{tabular}


Table 2 (continued)

\begin{tabular}{|c|c|c|c|c|c|c|c|c|c|}
\hline Biomarker & Sample & Demographic & $\begin{array}{l}\text { Specificity } \\
(\%)\end{array}$ & $\begin{array}{l}\text { Sensitivity } \\
(\%)\end{array}$ & Cut-off & $R^{2}$ & AUC & Clinical relevance & References \\
\hline \multirow[t]{3}{*}{ Zonulin } & Plasma & Sepsis $=25$ & - & - & $6.61 \mathrm{ng} / \mathrm{ml}$ & - & - & $\begin{array}{l}\uparrow \text { Zonulin sepsis com- } \\
\text { pared to post-surgical } \\
\text { and control groups, } \\
p=0.008\end{array}$ & [39] \\
\hline & & Post-surgical $=18$ & - & - & - & - & - & $\begin{array}{l}\text { No difference between } \\
\text { survivors and non- } \\
\text { survivors, } p=0.305\end{array}$ & \\
\hline & & Control $=20$ & - & - & - & - & $\begin{array}{l}0.01 \\
-0.46,- \\
0.19, \text { and } \\
0.10\end{array}$ & $\begin{array}{l}\uparrow \text { Zonulin, no correlation } \\
\text { with CRP, APACHE-II, } \\
\text { SAPSII, SOFA, } p=0.997 \text {, } \\
p=0.077, p=0.491 \text {, and } \\
p=0.671 \text {, subsequently }\end{array}$ & \\
\hline \multirow[t]{3}{*}{ D-lactic acid } & Serum & Sepsis $=30$ & - & - & $\begin{array}{l}15.32 \text { and } \\
27.95 \mathrm{mg} / \mathrm{l}\end{array}$ & - & - & $\begin{array}{l}\uparrow D \text {-lactic acid sepsis } \\
\text { and septic shock groups, } \\
p<0.01\end{array}$ & {$[40]$} \\
\hline & & Septic shock $=30$ & - & - & & - & - & $\begin{array}{l}\uparrow \mathrm{D} \text {-lactic acid is no dif- } \\
\text { ferent between survivors } \\
\text { and non-survivors }\end{array}$ & \\
\hline & & Control $=20$ & & & & & & & \\
\hline \multicolumn{10}{|c|}{ Non-coding RNAs } \\
\hline \multirow[t]{11}{*}{ Lnc-MALAT1 } & Plasma & Sepsis $=196$ & - & - & - & - & 0.866 & $\begin{array}{l}\uparrow \text { Lnc-MALAT1/miR-125a } \\
\text { axis in sepsis patients }\end{array}$ & [41] \\
\hline & & Age $=58.2 \pm 11.2$ & - & - & - & - & - & $\begin{array}{l}\uparrow \text { Lnc-MALAT1 relative } \\
\text { expression in sepsis } \\
\text { patients }\end{array}$ & \\
\hline & & Control $=196$ & - & - & - & - & - & $\begin{array}{l}\text { Lnc-MALAT1/miRNA- } \\
\text { 125a axis discriminates } \\
\text { sepsis patients from } \\
\text { healthy controls and } \\
\text { exhibits a positive } \\
\text { association with general } \\
\text { disease severity, organ } \\
\text { injury, inflammation } \\
\text { level, and mortality in } \\
\text { sepsis patients }\end{array}$ & \\
\hline & & Age $=57.1 \pm 12.1$ & & & & & & & \\
\hline & Plasma & Sepsis $=152$ & $68.50 \%$ & $65.90 \%$ & - & - & $\begin{array}{l}0,674 \\
\text { (ARDS }\end{array}$ & $\begin{array}{l}\uparrow \text { Inc-MALAT1 correlates } \\
\text { with raised ARDS risk, } \\
\text { disease severity, and } \\
\text { increased mortality in } \\
\text { septic patients }\end{array}$ & [42] \\
\hline & & Age $=59.7 \pm 11.2$ & $38.30 \%$ & $88.60 \%$ & - & - & 0.651 & $\begin{array}{l}\text { High mortality in sepsis } \\
\text { patients }\end{array}$ & \\
\hline & & & - & - & - & - & - & $\begin{array}{l}\text { Lnc-MALAT1 expression } \\
\text { was positively correlated } \\
\text { with inflammatory factor } \\
\text { levels (CRP, PCT, TNF-a, } \\
\text { IL-1 } \beta \text {, IL-6, and IL-17) in } \\
\text { septic patients }\end{array}$ & \\
\hline & Plasma & Sepsis $=120$ & - & - & - & - & 0.91 & $\begin{array}{l}\uparrow \text { Inc-MALAT1 in septic } \\
\text { patients, distinguishing } \\
\text { patients with sepsis from } \\
\text { control }\end{array}$ & [110] \\
\hline & & Control $=60$ & - & - & - & - & 0.836 & $\begin{array}{l}\text { Septic shock patients } \\
\text { compared to patients } \\
\text { without septic shock }\end{array}$ & \\
\hline & & & - & - & - & - & 0.886 & $\begin{array}{l}\text { Non-survivors compared } \\
\text { to surviving patients }\end{array}$ & \\
\hline & & & - & - & - & - & - & $\begin{array}{l}\uparrow \text { Lnc-MALAT1 expres- } \\
\text { sion was an independent } \\
\text { risk factor for sepsis, } \\
\text { septic shock, and poor } \\
\text { prognosis }\end{array}$ & \\
\hline
\end{tabular}


Table 2 (continued)

\begin{tabular}{|c|c|c|c|c|c|c|c|c|c|}
\hline Biomarker & Sample & Demographic & $\begin{array}{l}\text { Specificity } \\
\text { (\%) }\end{array}$ & $\begin{array}{l}\text { Sensitivity } \\
\text { (\%) }\end{array}$ & Cut-off & $R^{2}$ & AUC & Clinical relevance & References \\
\hline \multirow[t]{8}{*}{ Inc-MEG3 } & Plasma & Sepsis $=219$ & - & - & - & - & 0,887 & $\begin{array}{l}\uparrow \text { Inc-MEG3 expression } \\
\text { predicting elevated } \\
\text { sepsis risk }\end{array}$ & {$[43]$} \\
\hline & & Control $=219$ & - & - & - & - & 0.934 & $\begin{array}{l}\text { Inc-MEG3/miR-21 axis } \\
\text { predicting elevated } \\
\text { sepsis risk }\end{array}$ & \\
\hline & & Age $=56.5 \pm 10.3$ & - & - & - & - & 0.801 & $\begin{array}{l}\text { miR-21 was predicting } \\
\text { reduced sepsis risk }\end{array}$ & \\
\hline & & & - & - & - & - & 0.704 & $\begin{array}{l}\text { Inc-MEG3 predicting } \\
\text { 28-day mortality risk }\end{array}$ & \\
\hline & & & - & - & - & - & 0.669 & $\begin{array}{l}\text { Inc-MEG3/miR-21 axis } \\
\text { predicting 28-day mor- } \\
\text { tality risk }\end{array}$ & \\
\hline & & & - & - & - & - & - & $\begin{array}{l}\uparrow \text { Inc-MEG3/miR-21 axis, } \\
\text { while } \downarrow \text { miR-21 expres- } \\
\text { sion was decreased in } \\
\text { sepsis patients }\end{array}$ & \\
\hline & & & & & & & - & $\begin{array}{l}\text { Inc-MEG3 expression } \\
\text { and Inc-MEG3/miR-21 } \\
\text { axis positively cor- } \\
\text { related, whereas miR-21 } \\
\text { expression negatively } \\
\text { correlated with APACHE- } \\
\text { II, SOFA, and inflamma- } \\
\text { tory molecules in sepsis } \\
\text { patients }\end{array}$ & \\
\hline & & & & & & & & $\begin{array}{l}\uparrow \text { Inc-MEG3 rela- } \\
\text { tive expression and } \\
\text { Inc-MEG3/miR-21 axis } \\
\text { in deaths than that in } \\
\text { survivor }\end{array}$ & \\
\hline \multicolumn{10}{|l|}{ miRNA } \\
\hline \multirow[t]{6}{*}{$\begin{array}{l}\text { miR-125a, } \\
\text { miR-125b }\end{array}$} & Plasma & Sepsis $=120$ & - & - & - & - & 0.557 & $\begin{array}{l}\leftrightarrow \text { miR-125a expression } \\
\text { between groups of } \\
\text { patients and not dif- } \\
\text { ferentiate sepsis patients } \\
\text { from controls }\end{array}$ & [84] \\
\hline & & Control $=120$ & - & - & - & - & 0.658 & $\begin{array}{l}\uparrow \text { miR-125b in sepsis } \\
\text { patients and can dis- } \\
\text { tinguish sepsis patients } \\
\text { from control healths }\end{array}$ & \\
\hline & & $59.1 \pm 12.1$ & - & - & - & - & - & $\begin{array}{l}\text { Positive correlation } \\
\text { between miR-125a } \\
\text { and miR-125b in sepsis } \\
\text { patients and controls }\end{array}$ & \\
\hline & & & - & - & - & - & - & $\begin{array}{l}\text { miR-125a was not cor- } \\
\text { related with APACHE-Il } \\
\text { or SOFA score, while } \\
\text { miR-125b was positively } \\
\text { associated with both } \\
\text { scores }\end{array}$ & \\
\hline & & & - & - & - & - & - & $\begin{array}{l}\downarrow \text { miR-125b in survivors } \\
\text { compared with non- } \\
\text { survivors }\end{array}$ & \\
\hline & & & - & - & - & - & - & $\begin{array}{l}\uparrow \text { miR-125b, but not miR- } \\
125 a \text {, is correlated with } \uparrow \\
\text { disease severity, inflam- } \\
\text { mation, and } \uparrow \text { mortality } \\
\text { in sepsis patients }\end{array}$ & \\
\hline
\end{tabular}


Table 2 (continued)

\begin{tabular}{|c|c|c|c|c|c|c|c|c|c|}
\hline Biomarker & Sample & Demographic & $\begin{array}{l}\text { Specificity } \\
\text { (\%) }\end{array}$ & $\begin{array}{l}\text { Sensitivity } \\
\text { (\%) }\end{array}$ & Cut-off & $R^{2}$ & AUC & Clinical relevance & References \\
\hline & \multirow[t]{5}{*}{ Plasma } & Sepsis $=126$ & - & - & - & - & 0.817 & $\begin{array}{l}\downarrow \text { miR-125a good predic- } \\
\text { tive values for sepsis risk }\end{array}$ & [85] \\
\hline & & Control $=125$ & - & - & - & - & 0.843 & $\begin{array}{l}\uparrow \text { Inc-ANRIL/miR-125a } \\
\text { axis for sepsis risk }\end{array}$ & \\
\hline & & Age $=56.6 \pm 13$ & - & - & - & - & 0.745 & $\begin{array}{l}\downarrow \text { miR-125a expression } \\
\text { in deaths than those in } \\
\text { survivors }\end{array}$ & \\
\hline & & & - & - & - & - & 0.785 & $\begin{array}{l}\uparrow \text { Inc-ANRIL/miR-125a } \\
\text { differentiating deaths } \\
\text { from survivors }\end{array}$ & \\
\hline & & & - & - & - & - & - & $\begin{array}{l}\text { Inc-ANRIL/miR-125a axis } \\
\text { positively correlated, and } \\
\text { miR-125a was negatively } \\
\text { associated with disease } \\
\text { severity and inflamma- } \\
\text { tion in sepsis patients }\end{array}$ & \\
\hline & \multirow[t]{7}{*}{ Plasma } & Sepsis $=150$ & - & - & - & - & $\begin{array}{l}0.749 \text { and } \\
0.839\end{array}$ & $\begin{array}{l}\uparrow \text { miR-125a and miR- } \\
125 \mathrm{~b} \text { distinguish sepsis } \\
\text { patients from controls }\end{array}$ & [111] \\
\hline & & Age $=56.9 \pm 10.3$ & - & - & - & - & 0.588 & $\begin{array}{l}\text { miR-125a to predict } \\
\text { 28-day mortality risk }\end{array}$ & \\
\hline & & Control $=150$ & - & - & - & - & 0.699 & $\begin{array}{l}\text { miR-125b had a potential } \\
\text { value in predicting } \\
\text { elevated 28-day mortal- } \\
\text { ity risk }\end{array}$ & \\
\hline & & Age $=55.1 \pm 11.4$ & - & - & - & - & - & $\begin{array}{l}\text { miR-125a failed to pre- } \\
\text { dict the } 28 \text {-day mortality } \\
\text { risk in sepsis patients }\end{array}$ & \\
\hline & & & - & - & - & - & - & $\begin{array}{l}\text { 1. The predictive value of } \\
\text { miR-125b for sepsis risk }\end{array}$ & \\
\hline & & & - & - & - & - & & & \\
\hline & & & & & & & & $\begin{array}{l}\text { miR-125a and miR-125b } \\
\text { relative expressions were } \\
\text { positively associated } \\
\text { with disease severity in } \\
\text { sepsis patients }\end{array}$ & \\
\hline & \multirow[t]{4}{*}{ Plasma } & Sepsis $=196$ & - & - & - & - & - & $\begin{array}{l}\uparrow \text { Inc-MALAT1/miR-125a } \\
\text { axis in sepsis patients, } \\
p<0.001\end{array}$ & [41] \\
\hline & & Age $=58.2 \pm 11.2$ & - & - & - & - & 0.931 & $\begin{array}{l}\text { Inc-MALAT1/miRNA-125a } \\
\text { axis discriminates sepsis } \\
\text { patients from control }\end{array}$ & \\
\hline & & Control $=196$ & - & - & - & - & 0.866 & $\begin{array}{l}\text { Inc-MALAT1 discrimi- } \\
\text { nates sepsis patients } \\
\text { from control }\end{array}$ & \\
\hline & & Age $=57.1 \pm 12.1$ & & & & & & & \\
\hline
\end{tabular}


Table 2 (continued)

\begin{tabular}{|c|c|c|c|c|c|c|c|c|c|}
\hline Biomarker & Sample & Demographic & $\begin{array}{l}\text { Specificity } \\
(\%)\end{array}$ & $\begin{array}{l}\text { Sensitivity } \\
\text { (\%) }\end{array}$ & Cut-off & $R^{2}$ & AUC & Clinical relevance & References \\
\hline \multicolumn{10}{|c|}{ Membrane receptors, cell proteins, and metabolites } \\
\hline \multirow[t]{11}{*}{ CD64 } & \multirow[t]{7}{*}{ Blood } & Sepsis $=119$ & - & - & - & - & - & $\begin{array}{l}\uparrow n C D 64 \text { and SOFA score } \\
\text { in the sepsis compared } \\
\text { to control } p<0.05\end{array}$ & [46] \\
\hline & & Septic shock $=32$ & - & - & $\begin{array}{l}4.1,9 \text {, and } 2.2 \\
\mathrm{MFI}\end{array}$ & - & - & $\begin{array}{l}\uparrow \text { Sepsis and septic } \\
\text { shock compared to } \\
\text { control } p<0.001\end{array}$ & \\
\hline & & \multirow[t]{5}{*}{ Control $=20$} & - & - & - & - & 0.879 & $\begin{array}{l}\text { nCD64 in bacterial } \\
\text { infection }\end{array}$ & \\
\hline & & & - & - & - & - & 0.888 & $\begin{array}{l}\uparrow \text { AUC of nCD64 } \\
\text { combined with SOFA } \\
\text { than that of any other } \\
\text { parameter alone or in } \\
\text { combination }\end{array}$ & \\
\hline & & & - & - & - & - & 0.85 & $\begin{array}{l}\text { CD64 for predicting } \\
\text { death }\end{array}$ & \\
\hline & & & - & - & - & - & 0.916 & $\begin{array}{l}\text { Combination of nCD64 } \\
\text { and SOFA score }\end{array}$ & \\
\hline & & & - & - & $\begin{array}{l}4.1 \text { versus } 8.9 \\
\mathrm{MFI}\end{array}$ & - & - & $\begin{array}{l}\uparrow n C D 64 \text { survivors versus } \\
\text { non-survivors } p<0.001\end{array}$ & \\
\hline & \multirow[t]{4}{*}{ Blood } & Sepsis $=20$ & \multirow{4}{*}{$\begin{array}{l}0.82,0.67 \\
\text { and } 0.67\end{array}$} & \multirow{4}{*}{$\begin{array}{l}0.67,0.76 \\
\text { and } 0.76\end{array}$} & \multirow{4}{*}{$\begin{array}{l}<90.40,<3.01 \\
\text { and }<0.825\end{array}$} & \multirow[t]{4}{*}{-} & \multirow{4}{*}{$\begin{array}{l}0.843 \text {, } \\
0.824 \text {, and } \\
0.804\end{array}$} & \multirow{4}{*}{$\begin{array}{l}\uparrow C D 64, \downarrow C D 13, \text { and } \\
\downarrow \text { HLA-DR predict mortal- } \\
\text { ity in septic patients }\end{array}$} & \multirow[t]{4}{*}{ [45] } \\
\hline & & Age $=54.35 \pm 17.97$ & & & & & & & \\
\hline & & Control $=20$ & & & & & & & \\
\hline & & Age $=51.55 \pm 13.37$ & & & & & & & \\
\hline \multirow[t]{4}{*}{ CD68 } & \multirow[t]{4}{*}{ Brain } & Septic shock $=16$ & \multirow[t]{4}{*}{-} & \multirow[t]{4}{*}{-} & \multirow[t]{4}{*}{-} & \multirow[t]{4}{*}{-} & \multirow[t]{4}{*}{-} & \multirow{4}{*}{$\begin{array}{l}\uparrow C D 68 \text { in the hippocam- } \\
\text { pus ( } 1.5 \text { fold), putamen } \\
\text { ( } 2.2 \text { fold), and cerebellum } \\
\text { ( } 2.5 \text { fold) in patients } \\
\text { with sepsis than control } \\
\text { patients }\end{array}$} & \multirow[t]{4}{*}{ [86] } \\
\hline & & Age $=8.9-71.7$ & & & & & & & \\
\hline & & Control $=15$ & & & & & & & \\
\hline & & Age $=65.2-87.4$ & & & & & & & \\
\hline \multirow[t]{7}{*}{ NFL } & \multirow[t]{7}{*}{ CSF and plasma } & Sepsis $=20$ & - & - & $1723.4,1905.2$ & - & - & $\begin{array}{l}\text { Day } 1-\text { sepsis versus } \\
\text { control } p>0.05\end{array}$ & \multirow[t]{7}{*}[87]{} \\
\hline & & Age $=66.7 \pm 14.0$ & - & - & $2753.1,2208.0$ & - & - & $\begin{array}{l}\text { Day } 3 \text { - sepsis versus } \\
\text { control } p>0.05\end{array}$ & \\
\hline & & Control $=5$ & - & - & $\begin{array}{l}\text { 5309.6, } \\
3701.3 \mathrm{pg} / \mathrm{ml}\end{array}$ & - & - & $\begin{array}{l}\text { Day } 7-\text { sepsis versus } \\
\text { control } p>0.05\end{array}$ & \\
\hline & & Age $=61.2 \pm 24.7$ & - & - & - & - & - & $\begin{array}{l}\uparrow N F L \text { in patient septic } \\
\text { compared to control } \\
\text { from day } 1 p=0.0063\end{array}$ & \\
\hline & & & - & - & - & - & - & $\begin{array}{l}\uparrow N F L \text { patients with sep- } \\
\text { sis-associated encepha- } \\
\text { lopathy } p=0.011\end{array}$ & \\
\hline & & & - & - & - & - & - & $\begin{array}{l}\uparrow N F L \text { correlated with } \\
\text { the severity of sepsis- } \\
\text { associated encephalopa- } \\
\text { thy } p=0.022\end{array}$ & \\
\hline & & & - & - & & - & - & $\begin{array}{l}\uparrow N F L \text { at CSF in non- } \\
\text { survivors compared to } \\
\text { survivors } p=0.012\end{array}$ & \\
\hline \multirow[t]{4}{*}{ NFH } & \multirow[t]{4}{*}{ CSF and plasma } & Sepsis $=20$ & - & - & $17.6,100.3$ & - & - & $\begin{array}{l}\text { Day } 1-\text { sepsis versus } \\
\text { control } p>0.05\end{array}$ & \multirow[t]{4}{*}{ [87] } \\
\hline & & Age $=66.7 \pm 14$ & - & - & $18.9,163.1$ & - & - & $\begin{array}{l}\text { Day } 3 \text { - sepsis versus } \\
\text { control } p>0.05\end{array}$ & \\
\hline & & Control $=5$ & - & - & $164.3,519.9$ & - & - & $\begin{array}{l}\text { Day } 7-\text { sepsis versus } \\
\text { control } p=0.016\end{array}$ & \\
\hline & & Age $=61.2 \pm 24.7$ & - & - & $\mathrm{ng} / \mathrm{ml}$ & - & - & $\begin{array}{l}\uparrow N F H \text { from day } 1 \text { in sep- } \\
\text { tic patients } p=0.043\end{array}$ & \\
\hline
\end{tabular}


Table 2 (continued)

\begin{tabular}{|c|c|c|c|c|c|c|c|c|c|}
\hline Biomarker & Sample & Demographic & $\begin{array}{l}\text { Specificity } \\
(\%)\end{array}$ & $\begin{array}{l}\text { Sensitivity } \\
\text { (\%) }\end{array}$ & Cut-off & $R^{2}$ & AUC & Clinical relevance & References \\
\hline \multirow[t]{9}{*}{ NSE } & Serum & $\begin{array}{l}\text { Sepsis/ sepsis-associated } \\
\text { encephalopathy }=48\end{array}$ & - & - & $\begin{array}{l}24.87 \text { and } \\
15.49 \mathrm{ng} / \mathrm{ml}\end{array}$ & - & - & $\begin{array}{l}\uparrow \text { NSE in sepsis-associ- } \\
\text { ated encephalopathy } \\
\text { group versus no- sepsis- } \\
\text { associated encephalopa- } \\
\text { thy group } p=0.003\end{array}$ & [80] \\
\hline & & $\begin{array}{l}\text { Age }=56 \pm 16 \text { Sepsis/ } \\
\text { non- sepsis-associated } \\
\text { encephalopathy }=64\end{array}$ & & & $24.15 \mathrm{ng} / \mathrm{ml}$ & & & $\begin{array}{l}\text { Diagnostic of sepsis- } \\
\text { associated encepha- } \\
\text { lopathy }\end{array}$ & \\
\hline & & Age $=52 \pm 17$ & $82.80 \%$ & $54.20 \%$ & - & - & 0.664 & $\begin{array}{l}\leftrightarrow \text { NSE, sepsis-survivors } \\
\text { versus sepsis-non-survi- } \\
\text { vors } p=0.108\end{array}$ & \\
\hline & & & - & - & & - & - & & \\
\hline & Plasma & Sepsis $=124$ & - & - & $>12.5 \mathrm{ug} / \mathrm{l}$ & - & - & $\begin{array}{l}23.3 \% \text {, increased risk } \\
\text { of } 30-\text { day mortality, } \\
p=0.006 \text {, and a } 29.3 \% \\
\text { increased risk of delirium } \\
p=0.005\end{array}$ & {$[88]$} \\
\hline & & Mean age $=52-71$ & - & - & - & - & - & $\begin{array}{l}\uparrow N S E \text { is associated with } \\
\text { mortality } p=0.003 \text {, and } \\
\text { delirium in critically ill } \\
\text { septic patients } p<0.001\end{array}$ & \\
\hline & CSF and plasma & $\begin{array}{l}\text { Sepsis/ sepsis-associated } \\
\text { encephalopathy }=12\end{array}$ & - & - & $\begin{array}{l}\text { Eight versus } \\
3.8 \mathrm{ng} / \mathrm{ml}\end{array}$ & - & - & $\begin{array}{l}\uparrow \text { CSF NSE in sepsis } \\
\text { group compared to } \\
\text { controls } p<0.05\end{array}$ & [112] \\
\hline & & Control $=21$ & & & & & & $\begin{array}{l}\leftrightarrow \text { Plasma NSE sepsis } \\
\text { group versus control } \\
\text { group }\end{array}$ & \\
\hline & & Mean age $=67.8 \pm 12.1$ & - & - & - & - & - & & \\
\hline \multirow[t]{6}{*}{ Presepsin } & Blood & Sepsis $=33$ & $90.70 \%$ & $98.60 \%$ & $407 \mathrm{pg} / \mathrm{ml}$ & - & 0.954 & $\begin{array}{l}\uparrow \text { Presepsin in sepsis } \\
\text { patients compared to } \\
\text { SIRS group } p<0.05\end{array}$ & {$[48]$} \\
\hline & & Severe sepsis $=24$ & & & & & & $\begin{array}{l}\uparrow \text { Presepsin and } \\
\text { APACHE-II score in severe } \\
\text { sepsis group than sepsis } \\
\text { group } p<0.05\end{array}$ & \\
\hline & & Septic shock $=15$ & - & - & - & - & - & $\begin{array}{l}\uparrow \text { Presepsin } \\
\text { and APACHE-II score in } \\
\text { septic shock group com- } \\
\text { pared to severe sepsis } \\
\text { group } p<0.05\end{array}$ & \\
\hline & & $\mathrm{SIRS}=23$ & & & & & & & \\
\hline & & Normal $=20$ & - & - & - & - & - & & \\
\hline & & Mean age $=62.1$ & & & & & & & \\
\hline \multirow[t]{8}{*}{ TREM-1 } & Serum & Severe sepsis $=34$ & - & - & $\begin{array}{l}129 \mathrm{pg} / \mathrm{ml} \\
\text { versus } 105 \mathrm{pg} / \\
\mathrm{ml}\end{array}$ & - & - & $\begin{array}{l}\uparrow \text { TREM-1 levels in septic } \\
\text { shock compared to } \\
\text { severe sepsis }\end{array}$ & [89] \\
\hline & & Septic shock $=53$ & - & - & - & - & - & $\begin{array}{l}\leftrightarrow \text { TREM-1 did not differ- } \\
\text { entiate between septic } \\
\text { shock and severe sepsis }\end{array}$ & \\
\hline & & Age $=2$ mo to 16 years & $56 \%$ & $60 \%$ & $116.47 \mathrm{pg} / \mathrm{ml}$ & - & 0.62 & Predict septic shock & \\
\hline & & & $52 \%$ & $71 \%$ & $116.47 \mathrm{pg} / \mathrm{ml}$ & - & 0.63 & Predict mortality & \\
\hline & & & - & - & - & - & - & $\begin{array}{l}\leftrightarrow \text { TREM-1 non-survivors } \\
\text { versus survivors }\end{array}$ & \\
\hline & Serum & $\mathrm{SIRS}=38$ & $73.30 \%$ & $71.10 \%$ & $\geq 133 \mathrm{pg} / \mathrm{ml}$ & - & - & sTREM-1 cut-off for sepsis & {$[113]$} \\
\hline & & Sepsis $=52$ & - & - & - & - & - & $\begin{array}{l}\uparrow \text { sTREM-1 in sepsis } \\
\text { group } p=0.001\end{array}$ & \\
\hline & & Age $=20$ to 92 & - & - & - & - & - & $\begin{array}{l}\uparrow \text { sTREM- } 1 \text { in the patients } \\
\text { with positive blood } \\
\text { culture } p=0.002\end{array}$ & \\
\hline
\end{tabular}


Table 2 (continued)

\begin{tabular}{|c|c|c|c|c|c|c|c|c|c|}
\hline Biomarker & Sample & Demographic & $\begin{array}{l}\text { Specificity } \\
\text { (\%) }\end{array}$ & $\begin{array}{l}\text { Sensitivity } \\
\text { (\%) }\end{array}$ & Cut-off & $R^{2}$ & AUC & Clinical relevance & References \\
\hline & $\begin{array}{l}\text { Plasma and } \\
\text { leukocytes }\end{array}$ & $\begin{array}{l}\text { Septic shock }=60 \text { Post- } \\
\text { operative }=30\end{array}$ & $100 \%$ & $98.30 \%$ & $30.0 \mathrm{pg} / \mathrm{ml}$ & - & - & $\begin{array}{l}\uparrow \text { sTREM-1 plasma in } \\
\text { septic shock compared } \\
\text { to control and postop- } \\
\text { erative groups } p<0.05\end{array}$ & [91] \\
\hline & & Control $=30$ & - & - & - & - & - & $\begin{array}{l}\uparrow \text { sTREM- } 1 \text { compared } \\
\text { with postoperative } \\
\text { group } p<0.05\end{array}$ & \\
\hline & & & - & - & - & - & 0.955 & $\begin{array}{l}\uparrow \text { TREM-1 expression on } \\
\text { human monocytes of a } \\
\text { septic shock compared } \\
\text { to control and postop- } \\
\text { erative groups } p<0.05\end{array}$ & \\
\hline \multicolumn{10}{|c|}{ Peptide precursor of the hormone and hormone } \\
\hline \multirow[t]{4}{*}{ MR-proADM } & Plasma & $\begin{array}{l}\text { Sepsis/bacterial iso- } \\
\text { late }=39\end{array}$ & $78 \%$ & $74.20 \%$ & $\geq 1.5$ & - & 0.82 & $\begin{array}{l}\uparrow \text { MR-proADM sepsis } \\
\text { versus control } p<0.0001\end{array}$ & [92] \\
\hline & & $\begin{array}{l}\text { Sepsis w/bacterial } \\
\text { isolate }=23\end{array}$ & $80 \%$ & $89.36 \%$ & $\geq 1.70$ & - & 0.92 & $\begin{array}{l}\uparrow \text { MR-proADM septic } \\
\text { shock versus control } \\
p<0.0001\end{array}$ & \\
\hline & & Septic shock $=47$ & $77.40 \%$ & $59.60 \%$ & $>3.00$ & - & 0.7 & $\begin{array}{l}\uparrow M R-p r o A D M \text { septic } \\
\text { shock versus sepsis } \\
p<0.0001\end{array}$ & \\
\hline & & Control $=50$ & - & - & $\begin{array}{l}4.37 \text { versus } \\
2.34 \mathrm{nmol} / \mathrm{l}\end{array}$ & - & - & $\begin{array}{l}\uparrow M R-\text { proADM, non- } \\
\text { survivor versus survivor } \\
p<0.0001\end{array}$ & \\
\hline \multirow[t]{10}{*}{ Bio-ADM } & & Sepsis $=632$ & - & - & - & & - & $\begin{array}{l}\text { Median sepsis } \\
\text { patients }=74 \mathrm{pg} / \mathrm{mL} \text {; } \\
\text { septic shock }=107 \mathrm{pg} / \\
\mathrm{mL} \text {, and } 29 \mathrm{pg} / \mathrm{mL} \text { in } \\
\text { non-septic patients }\end{array}$ & [53] \\
\hline & & Septic shock $=267$ & - & - & - & & & $\begin{array}{l}\text { Mortality in sepsis } \\
\text { patients OR of } 1.23\end{array}$ & \\
\hline & & Non-septic $=1235$ & - & - & - & & - & $\begin{array}{l}\uparrow \text { Dialysis: OR } 1.97 \text { in } \\
\text { sepsis patients }\end{array}$ & \\
\hline & & & - & - & $70 \mathrm{pg} / \mathrm{mL}$ & & & $\begin{array}{l}\uparrow \text { bio-ADM } \uparrow \text { Use of } \\
\text { vasopressors, OR } 1.33\end{array}$ & \\
\hline & & & - & - & $108 \mathrm{pg} / \mathrm{mL}$ & & - & $\begin{array}{l}\text { Survivors and non- } \\
\text { survivors in sepsis }\end{array}$ & \\
\hline & & & - & - & - & & & $\begin{array}{l}\text { Youden's index derived } \\
\text { threshold of performed } \\
\text { better }\end{array}$ & \\
\hline & & & - & - & & & - & $\uparrow$ bio-ADM non-survivors & \\
\hline & & & & & & & - & & \\
\hline & & & & & & & - & & \\
\hline & & & & & & & - & & \\
\hline \multirow[t]{6}{*}{ PCT } & Serum & Sepsis $=59$ & - & - & - & - & - & $\uparrow P C T p<0.0005$ & {$[66]$} \\
\hline & & $\begin{array}{l}\text { Severe sepsis/septic } \\
\text { shock }=71\end{array}$ & - & - & $\begin{array}{l}0.67 \text { versus } \\
3.81\end{array}$ & - & - & $\begin{array}{l}\text { Survivor versus non- } \\
\text { survivor at seven days }\end{array}$ & \\
\hline & & Mean age $=80$ & - & - & $\begin{array}{l}0.48 \text { versus } \\
1.82 \mathrm{ng} / \mathrm{mL}\end{array}$ & - & - & $\begin{array}{l}\text { Survivor versus non- } \\
\text { survivor at } 30 \text { days }\end{array}$ & \\
\hline & Serum & $\mathrm{SIRS}=38$ & $65.79 \%$ & $67.33 \%$ & $1.57 \mathrm{ng} / \mathrm{ml}$ & - & - & PCT cut-off for sepsis & [113] \\
\hline & & Sepsis $=52$ & - & - & - & - & - & $\begin{array}{l}\uparrow P C T \text { in sepsis group, } \\
p=0.01\end{array}$ & \\
\hline & & Age $=20$ to 92 & & & & & & & \\
\hline
\end{tabular}


Table 2 (continued)

\begin{tabular}{|c|c|c|c|c|c|c|c|c|c|}
\hline Biomarker & Sample & Demographic & $\begin{array}{l}\text { Specificity } \\
\text { (\%) }\end{array}$ & $\begin{array}{l}\text { Sensitivity } \\
\text { (\%) }\end{array}$ & Cut-off & $R^{2}$ & AUC & Clinical relevance & References \\
\hline & \multirow[t]{4}{*}{ Serum } & Sepsis $=79$ & - & - & - & - & - & $\begin{array}{l}\uparrow P C T \text { concentrations in } \\
\text { patients with sepsis and } \\
\text { infection }\end{array}$ & \multirow[t]{4}{*}{ [114] } \\
\hline & & Age $=$ newborn to 12 & & & & & & $\begin{array}{l}\downarrow P C T \text { concentrations } \\
\text { with antibiotic treatment }\end{array}$ & \\
\hline & & Control $=21$ & - & - & & - & - & & \\
\hline & & Age $=$ newborn to 10 & & & & & & & \\
\hline & \multirow[t]{3}{*}{ Blood } & Sepsis $=119$ & - & - & $\begin{array}{l}17.1,1.8, \text { and } \\
0.04 \mathrm{ng} / \mathrm{ml}\end{array}$ & - & - & $\begin{array}{l}\uparrow P C T \text { septic shock and } \\
\text { sepsis compared to the } \\
\text { control group } p<0.001\end{array}$ & \multirow[t]{3}{*}{ [46] } \\
\hline & & Septic shock $=32$ & - & - & $\begin{array}{l}1.8 \text { and } 9.2 \mathrm{ng} / \\
\mathrm{ml}\end{array}$ & - & - & $\begin{array}{l}\uparrow P C T \text { levels in survivors } \\
\text { versus non-survivors } \\
p>0.001\end{array}$ & \\
\hline & & Control $=20$ & & & & & & & \\
\hline & \multirow[t]{6}{*}{ Blood } & Sepsis $=33$ & $90.70 \%$ & $98.60 \%$ & $407 \mathrm{pg} / \mathrm{ml}$ & - & 0.874 & $\begin{array}{l}\uparrow P C T \text { sepsis patients } \\
\text { compared to SIRS group } \\
p<0.05\end{array}$ & \multirow[t]{6}{*}{ [48] } \\
\hline & & Severe sepsis $=24$ & - & - & - & - & - & $\begin{array}{l}\uparrow P C T \text { and APACHEll } \\
\text { score in severe sepsis } \\
\text { group compared to } \\
\text { sepsis group } p<0.05\end{array}$ & \\
\hline & & Septic shock $=15$ & & & & & & & \\
\hline & & $\mathrm{SIRS}=23$ & & & & & & & \\
\hline & & Normal $=20$ & & & & & & & \\
\hline & & Mean age $=62.1$ & & & & & & & \\
\hline & \multirow[t]{3}{*}{ Plasma } & $\begin{array}{l}\text { Sepsis and shock sep- } \\
\text { tic }=1089\end{array}$ & & & & & & $\begin{array}{l}\leftrightarrow \text { There was no statistic } \\
\text { difference in the primary } \\
\text { outcome regarding PCT- } \\
\text { guidance } 27.9 \% \text { versus } \\
\text { no PCT-guidance } 22.9 \% \\
\text { to predict mortality } \\
p=0.18\end{array}$ & \multirow[t]{3}{*}{ [61] } \\
\hline & & PCT-guidance $n=279$ & & & & & & $\begin{array}{l}\leftrightarrow P C T \text {-guidance } \\
\text { versus no PCT-guidance } \\
\text { there was no statistic } \\
\text { difference in } 28 \text {-day } \\
\text { mortality, } 25.6 \% \text { versus } \\
28.2 \% p=0.34\end{array}$ & \\
\hline & & No PCT-guidance $n=267$ & & & & & & & \\
\hline & \multirow[t]{3}{*}{ Serum } & Severe sepsis $=34$ & - & - & \multirow{3}{*}{$\begin{array}{l}129 \mathrm{pg} / \mathrm{ml} \\
\text { versus } 105 \mathrm{pg} / \\
\mathrm{ml}\end{array}$} & \multirow[t]{3}{*}{-} & \multirow[t]{3}{*}{-} & \multirow{3}{*}{$\begin{array}{l}\leftrightarrow P C T \text { did not differenti- } \\
\text { ate septic shock from } \\
\text { severe sepsis }\end{array}$} & \multirow[t]{3}{*}{ [89] } \\
\hline & & Septic shock $=53$ & & & & & & & \\
\hline & & Age $=2 \mathrm{mo}$ to 16 years & & & & & & & \\
\hline \multirow[t]{4}{*}{ NT-proBNP } & \multirow[t]{4}{*}{ Serum } & Sepsis $=60$ & - & - & $1.209 \mathrm{ng} / \mathrm{l}$ & - & - & $\begin{array}{l}\uparrow N T \text {-proBNP level } \\
\text { at } 24 \mathrm{~h} \text { after sepsis } \\
\text { diagnosis }\end{array}$ & \multirow[t]{4}{*}{ [55] } \\
\hline & & Severe sepsis $=89$ & - & - & - & - & - & $\begin{array}{l}\uparrow N T \text {-proBNP levels } \\
\text { at } 24 \mathrm{~h} \text { after sepsis } \\
\text { onset were associated } \\
\text { with } \downarrow \text { SPPB scores at } \\
12 \text { months } p<0.05 \text {, and } \\
\downarrow \text { handgrip strength at } \\
\text { six and } 12 \text {-month follow- } \\
\text { up } p<0.001\end{array}$ & \\
\hline & & Septic shock $=47$ & & & & & & & \\
\hline & & Age $=59.1 \pm 15.1$ & & & & & & & \\
\hline
\end{tabular}


Table 2 (continued)

\begin{tabular}{|c|c|c|c|c|c|c|c|c|c|}
\hline Biomarker & Sample & Demographic & $\begin{array}{l}\text { Specificity } \\
\text { (\%) }\end{array}$ & $\begin{array}{l}\text { Sensitivity } \\
\text { (\%) }\end{array}$ & Cut-off & $R^{2}$ & AUC & Clinical relevance & References \\
\hline & Plasma & Sepsis $=142$ & & & $\begin{array}{l}4(2.6-8.8) \text { ver- } \\
\text { sus } 8.2 \mathrm{nmol} / \mathrm{L} \\
(5.2-12.6)\end{array}$ & & - & $\begin{array}{l}\uparrow N T \text {-proBNP levels in } \\
\text { non-survivors com- } \\
\text { pared with survivors } \\
p<0.01 \text {. } \leftrightarrow \text { CRP did not } \\
\text { change in survivors and } \\
\text { non-survivors }\end{array}$ & [52] \\
\hline & & Septic shock $=947$ & & & & & - & $\begin{array}{l}\uparrow N T \text {-proBNP prediction } \\
\text { of } 28 \text {-day mortality in } \\
\text { total population, sepsis } \\
\text { group, and shock septic } \\
\text { group, respectively }\end{array}$ & \\
\hline & & & & & & & $\begin{array}{l}0.73,0.73 \\
\text { and } 0.72\end{array}$ & & \\
\hline \multicolumn{10}{|c|}{ Neutrophil, cells, and related biomarkers } \\
\hline \multirow[t]{14}{*}{ Lactate } & Plasma & Sepsis $=59$ & - & - & - & & - & $\uparrow$ Lactate $p<0.0005$ & {$[66]$} \\
\hline & & $\begin{array}{l}\text { Severe sepsis/septic } \\
\text { shock }=71\end{array}$ & - & - & 1.7 versus 3.4 & & - & $\begin{array}{l}\text { Survivor versus non- } \\
\text { survivor at seven days }\end{array}$ & \\
\hline & & Mean age $=80$ & - & - & 1.6 versus 2.2 & & - & $\begin{array}{l}\text { Survivor versus non- } \\
\text { survivor at } 30 \text { days }\end{array}$ & \\
\hline & & & - & - & $\mathrm{mmol} / \mathrm{l}$ & & $\begin{array}{l}0.79 \text { and } \\
0.77\end{array}$ & $\begin{array}{l}\text { Predictors of mortality at } \\
7 \text { and } 30 \text { days } p=0.001\end{array}$ & \\
\hline & Serum & $\begin{array}{l}\text { Non- sepsis-associated } \\
\text { encephalopathy }=2513 \\
\text { Sepsis-associated } \\
\text { encephalopathy }=2474\end{array}$ & - & - & - & - & - & $\begin{array}{l}\uparrow \text { Lactate predicted } \\
30 \text {-day mortality of } \\
\text { patients with sepsis-asso- } \\
\text { ciated encephalopathy, } \\
\text { OR: } 1.19 p<0.0005\end{array}$ & [93] \\
\hline & Blood & Sepsis $=33$ & $90.70 \%$ & $98.60 \%$ & $407 \mathrm{pg} / \mathrm{ml}$ & - & $\begin{array}{l}0.859 \text { and } \\
0.723\end{array}$ & $\begin{array}{l}\uparrow \text { Lactate and APACHE-II } \\
\text { score in severe sepsis } \\
\text { group compared to } \\
\text { sepsis group } p<0.05\end{array}$ & [48] \\
\hline & & Severe sepsis $=24$ & - & - & - & - & - & $\begin{array}{l}\uparrow \text { APACHE-II score and } \\
\text { lactate in septic shock } \\
\text { group when compared } \\
\text { with severe sepsis group } \\
p<0.05\end{array}$ & \\
\hline & & Septic shock $=15$ & & & & & & & \\
\hline & & $\mathrm{SIRS}=23$ & & & & & & & \\
\hline & & Normal $=20$ & & & & & & & \\
\hline & & Mean age $=62.1$ & & & & & & & \\
\hline & Serum & Severe sepsis $=34$ & - & - & - & - & - & $\leftrightarrow$ Lactate did not dif- & [89] \\
\hline & & Septic shock $=53$ & & & & & & ferentiate septic shock & \\
\hline & & Age $=2$ mo to 16 years & & & & & & & \\
\hline \multirow[t]{2}{*}{ MPO } & Plasma & Sepsis $=957$ & - & - & $128.1 \mathrm{ng} / \mathrm{ml}$ & - & - & $\begin{array}{l}\uparrow \mathrm{MPO} \text { day } 1 \text { and } \\
\text { progressively decreased } \\
\text { until day } 7\end{array}$ & [94] \\
\hline & & & - & - & - & - & - & $\begin{array}{l}\uparrow M P O \text { increase on days } \\
\text { on days } 1,2 \text {, and } 7 \text { in } \\
90 \text {-day non-survivors } \\
p<0.003, p=0.03 \text {, and } \\
p=0.001\end{array}$ & \\
\hline
\end{tabular}


Table 2 (continued)

\begin{tabular}{|c|c|c|c|c|c|c|c|c|c|}
\hline Biomarker & Sample & Demographic & $\begin{array}{l}\text { Specificity } \\
\text { (\%) }\end{array}$ & $\begin{array}{l}\text { Sensitivity } \\
\text { (\%) }\end{array}$ & Cut-off & $R^{2}$ & AUC & Clinical relevance & References \\
\hline & & Septic shock $=55$ & - & - & - & - & - & $\begin{array}{l}\uparrow \text { MPO-DNA and cf-DNA } \\
\text { in patients with septic } \\
\text { shock on day } 1 p<0.01\end{array}$ & [95] \\
\hline & & Control $=13$ & - & - & - & - & - & $\begin{array}{l}\uparrow \text { MPO-DNA on days } \\
3 \text { and } 7 \text { of sepsis was } \\
\text { associated with } 28 \text {-day } \\
\text { mortality } p<0.01\end{array}$ & \\
\hline & & Mean age $=68$ & - & - & - & $\begin{array}{l}0.303 \\
\text { and } \\
0.434\end{array}$ & - & $\begin{array}{l}\uparrow \text { MPO-DNA on day } 3 \\
\text { and } 7 \text { positive correlation } \\
\text { with SOFA score } p=0.04 \\
\text { and } p=0.03 \text {, subse- } \\
\text { quently }\end{array}$ & \\
\hline & & $\delta=71 \%$ & & & & & & & \\
\hline \multirow[t]{13}{*}{ Resistin } & Plasma & Sepsis $=957$ & - & - & $192.9 \mathrm{ng} / \mathrm{ml}$ & - & - & $\begin{array}{l}\uparrow \text { Resistin on day one } \\
\text { and progressively } \\
\text { decreased until day } 7\end{array}$ & [94] \\
\hline & & Mean age $=70$ & - & - & - & - & - & $\begin{array}{l}\uparrow \text { Resistin increase on } \\
\text { days } 1,2 \text {, and } 7 \text { in } 90 \text {-day } \\
\text { non-survivors } p<0.001\end{array}$ & \\
\hline & & $\delta=60 \%$ & & & & & & & \\
\hline & Serum & Sepsis $=50$ & $\begin{array}{l}72 \%, 80 \% \\
\text { and } 100 \%\end{array}$ & $\begin{array}{l}82 \%, 95 \% \\
\text { and } 100 \%\end{array}$ & $\begin{array}{l}5.2,6.1, \text { and } \\
7,5 \mathrm{ng} / \mathrm{ml}\end{array}$ & - & - & $\begin{array}{l}\uparrow \text { Resistin levels on day } \\
1,4 \text {, and } 7\end{array}$ & {$[115]$} \\
\hline & & $\begin{array}{l}\text { Patient without sep- } \\
\text { sis }=22\end{array}$ & - & - & - & - & $\begin{array}{l}0.864 \text {, } \\
0.987 \text {, and } \\
0.987\end{array}$ & $\begin{array}{l}\uparrow \text { Resistin levels on days } \\
1,4 \text {, and } 7 \text { were associ- } \\
\text { ated with sepsis }\end{array}$ & \\
\hline & & Control $=25$ & & & & & & & \\
\hline & & Age $\leq 12$ & & & & & & & \\
\hline & Serum & Sepsis $=60$ & - & - & 36.45 & - & - & $\begin{array}{l}\uparrow \text { Resistin in sepsis/septic } \\
\text { shock groups } p=0.001\end{array}$ & {$[96]$} \\
\hline & & Septic shock $=42$ & - & - & $\begin{array}{l}48.13 \text { versus } \\
31.58\end{array}$ & - & - & $\begin{array}{l}\uparrow \text { Resistin levels in non- } \\
\text { survivors versus Survivors } \\
\text { on day } 1 \text { and } 7 p<0.001 \\
\text { and } p<0.001\end{array}$ & \\
\hline & & Control $=102$ & - & - & $\begin{array}{l}46.20 \text { versus } \\
25.22\end{array}$ & - & - & $\begin{array}{l}\uparrow \text { Resistin septic shock } \\
\text { versus sepsis on day } \\
1 \text { and } 3 p<0.001 \text { and } \\
p<0.001\end{array}$ & \\
\hline & & & & & $\begin{array}{l}40.8 \text { versus } \\
33.4\end{array}$ & & & & \\
\hline & & & & & $\begin{array}{l}37.1 \text { versus } \\
27.4\end{array}$ & & & & \\
\hline & & & & & $\mu \mathrm{g} / \mathrm{l}$ & & & & \\
\hline \multicolumn{10}{|c|}{ Soluble receptors } \\
\hline \multirow[t]{4}{*}{ SPD-L1 } & Serum & Sepsis $=483$ & - & - & $0.16 \mathrm{ng} / \mathrm{ml}$ & - & - & $\begin{array}{l}\uparrow \text { sPD-L1 immunosup- } \\
\text { pression phenotype, } \uparrow \\
\text { risk of hospital readmis- } \\
\text { sion and mortality, } \\
\mathrm{OR}=8.26\end{array}$ & {$[22]$} \\
\hline & & Mean age $=60.5$ & & & & & & $\begin{array}{l}\uparrow \text { sPD-L1, } 45(46.4 \%) \text { at } \\
3 \text { months, } 40(44.9 \%) \text { at } \\
6 \text { months, and } 44(49.4 \%) \\
\text { at } 12 \text { months }\end{array}$ & \\
\hline & & ○ $54.9 \%$ & - & - & - & - & - & $\begin{array}{l}\uparrow \text { sPD-L1 to predict } \\
28 \text {-day mortal- } \\
\text { ity } \cong \text { APACHE-II and SOFA } \\
\text { scores }\end{array}$ & \\
\hline & & & - & - & - & - & - & & \\
\hline
\end{tabular}


Table 2 (continued)

\begin{tabular}{|c|c|c|c|c|c|c|c|c|c|}
\hline Biomarker & Sample & Demographic & $\begin{array}{l}\text { Specificity } \\
\text { (\%) }\end{array}$ & $\begin{array}{l}\text { Sensitivity } \\
(\%)\end{array}$ & Cut-off & $R^{2}$ & AUC & Clinical relevance & References \\
\hline & Serum & Sepsis $=91$ & - & - & $2.09 \mathrm{ng} / \mathrm{ml}$ & - & - & $\begin{array}{l}\uparrow \text { SPD-L1 and SPD-1 } \\
\text { in septic patients } \\
p=0.0001\end{array}$ & [68] \\
\hline & & Control $=29$ & - & - & - & - & - & $\begin{array}{l}\uparrow \text { sPD-L1 increased in } \\
\text { non-survivors } p<0.05\end{array}$ & \\
\hline & & & - & - & - & - & 0.71 & $\begin{array}{l}\uparrow \text { sPD-L1 level to predict } \\
\text { 28-day mortality }\end{array}$ & \\
\hline \multirow[t]{8}{*}{ SUPAR } & Serum & Sepsis $=59$ & - & - & - & - & - & $\uparrow \operatorname{suPAR}, p<0.0005$ & [66] \\
\hline & & $\begin{array}{l}\text { Severe sepsis/septic } \\
\text { shock }=71\end{array}$ & - & - & 6.9 versus 9.8 & - & - & $\begin{array}{l}\text { Survivor versus non- } \\
\text { survivor at seven days }\end{array}$ & \\
\hline & & Mean age $=80$ & - & - & 6.4 versus 9.3 & - & - & $\begin{array}{l}\text { Survivor versus non- } \\
\text { survivor at } 30 \text { days }\end{array}$ & \\
\hline & & & - & - & $\mathrm{ng} / \mathrm{ml}$ & & $\begin{array}{l}0.72 \text { and } \\
0.77\end{array}$ & $\begin{array}{l}\text { Predictors of mortality at } \\
7 \text { and } 30 \text { days } p=0.006\end{array}$ & \\
\hline & & & - & - & - & - & - & $\begin{array}{l}\downarrow \text { suPAR from day } 1 \text { to } \\
\text { day seven sepsis and } \\
\text { severe sepsis/septic } \\
\text { shock } p<0.0005\end{array}$ & \\
\hline & Serum & Sepsis $=60$ & - & - & 13 & - & - & $\begin{array}{l}\uparrow \text { suPAR in sepsis and } \\
\text { septic shock }\end{array}$ & [96] \\
\hline & & Septic shock $=42$ & - & - & $\begin{array}{l}10.5 \text { versus } \\
14.1\end{array}$ & - & - & $\begin{array}{l}\uparrow \text { suPAR in septic shock } \\
\text { compared with sepsis on } \\
\text { day one but not on day } \\
7 p<0.04 \text { and } p=0.68 \text {, } \\
\text { subsequently }\end{array}$ & \\
\hline & & Control $=102$ & & & $\begin{array}{l}11.3 \text { versus } \\
12.9 \mu \mathrm{g} / \mathrm{l}\end{array}$ & & & & \\
\hline \multirow[t]{12}{*}{ sTNFR-1 } & Plasma & $\mathrm{SIRS}=943$ & - & - & $\begin{array}{l}7719 \text { versus } \\
18,197\end{array}$ & - & - & $\begin{array}{l}\uparrow \text { sTNFR-1 in non- } \\
\text { survivor versus survivor, } \\
p<0.001\end{array}$ & [81] \\
\hline & & Sepsis $=330$ & - & - & $\mathrm{pg} / \mathrm{ml}$ & - & - & $\begin{array}{l}\uparrow \text { sTNFR- } 1 \text { sepsis com- } \\
\text { pared to SIRS } p<0.001\end{array}$ & \\
\hline & & Shock $=216$ & & & & & & & \\
\hline & & Pneumonia $=169$ & & & & & & & \\
\hline & & Others $=152$ & & & & & & & \\
\hline & & Age $=55.1 \pm 16.1$ & & & & & & & \\
\hline & & đ $63.9 \%$ & & & & & & & \\
\hline & Plasma & No delirium $=47$ & - & - & $\begin{array}{l}3.843 \text { and } \\
10.250 \mathrm{pg} / \mathrm{ml}\end{array}$ & - & - & $\begin{array}{l}\uparrow \text { sTNFR1 and sTNFR2 } \\
\text { delirium cutoff } p=0.005\end{array}$ & [98] \\
\hline & & Delirium $=31$ & - & - & - & - & - & $\begin{array}{l}\uparrow \text { STNFR1 and sTNFR2 } \\
\text { in delirium group com- } \\
\text { pared with non-delirium } \\
p=0.005 \text {, and } p=0.003 \text {, } \\
\text { subsequently }\end{array}$ & \\
\hline & & & & & & & & $\begin{array}{l}\text { OR: } 18 \text { to sTNFR1, } \\
p=0.004 \text { and OR: } 51 \text { to }\end{array}$ & \\
\hline & & & & & & & & STNFR2, $p=0.006$ & \\
\hline & & & - & - & - & - & - & & \\
\hline
\end{tabular}


Table 2 (continued)

\begin{tabular}{|c|c|c|c|c|c|c|c|c|c|}
\hline Biomarker & Sample & Demographic & $\begin{array}{l}\text { Specificity } \\
\text { (\%) }\end{array}$ & $\begin{array}{l}\text { Sensitivity } \\
(\%)\end{array}$ & Cut-off & $R^{2}$ & AUC & Clinical relevance & References \\
\hline \multicolumn{10}{|l|}{ Lipoproteins } \\
\hline \multirow[t]{3}{*}{ LDL } & Serum & Sepsis $=594$ & - & - & - & - & - & $\begin{array}{l}\text { Risk of sepsis, OR, } 0.86 \text {, } \\
p=0.001 \text { and admission } \\
\text { to the ICU, OR, } 0.85 ; \\
p=0.008 ; \text { but not hospi- } \\
\text { tal mortality, OR, }\end{array}$ & [99] \\
\hline & & & & & & & & $\begin{array}{l}\downarrow \text { Quartile greater risk } \\
\text { of sepsis; OR, } 1.48 \text {; and } \\
\text { admission to the ICU, } \\
\text { OR, } 1.45 \text {, versus highest } \\
\text { quartile }\end{array}$ & \\
\hline & & & & & & & & $\begin{array}{l}\leftrightarrow \text { When comorbidities } \\
\text { were considered }\end{array}$ & \\
\hline \multirow[t]{9}{*}{ HDL } & Serum & Sepsis $=63$ & - & - & - & - & - & $\begin{array}{l}\downarrow \text { HDL in non-survivors } \\
\text { on days } 1 \text { to } 4\end{array}$ & [100] \\
\hline & & Mean age $=72$ & - & - & - & - & 0.84 & $\begin{array}{l}\text { Predicts mortality within } \\
30 \text { days }\end{array}$ & \\
\hline & & & $80 \%$ & $92 \%$ & $20 \mathrm{mg} / \mathrm{dl}$ & - & - & $\begin{array}{l}83 \% \text { accuracy to predict } \\
30 \text {-day overall mortality }\end{array}$ & \\
\hline & & & - & - & - & - & - & $\begin{array}{l}\mathrm{HDL}<20 \mathrm{mg} / \mathrm{dl} \text { increases } \\
\text { attributable mortality, } \\
\text { risk of prolonged ICU } \\
\text { stay, and hospital- } \\
\text { acquired infection rate }\end{array}$ & \\
\hline & Plasma & Suspected sepsis $=200$ & 0.690 & 0.716 & $30.9 \mathrm{mg} / \mathrm{dl}$ & - & 0,749 & MODS predictor & [101] \\
\hline & & & 0.699 & 0.857 & $25,1 \mathrm{mg} / \mathrm{dl}$ & - & 0,818 & Mortality in 28 days & \\
\hline & & & - & - & $<25.1 \mathrm{mg} / \mathrm{dl}$ & - & - & $\begin{array}{l}\uparrow \text { Mortality, } p<0.0001 \text { in } \\
28 \text { days and } p=0.0007 \\
\text { in } 90 \text { days }\end{array}$ & \\
\hline & & & - & - & - & - & - & $\begin{array}{l}74 \% \text { of patients with } \\
\text { HDL < } 25.1 \mathrm{mg} / \mathrm{dl} \\
\text { required ICU compared } \\
\text { to } 35 \% \text { above cutoff; } \\
\text { development of severe } \\
\text { acute renal dysfunction } \\
\text { was } 47 \% \text { versus } 21 \% \text {, } \\
\text { respectively; multiple } \\
\text { organ dysfunction was } \\
60 \% \text { versus } 25 \% \text {; and } \\
\text { mechanical ventilation } \\
\text { was } 53 \% \text { versus } 21 \%\end{array}$ & \\
\hline & & & - & - & - & - & - & $\begin{array}{l}\downarrow \mathrm{HD} \text {, the } 28 \text {-day mortal- } \\
\text { ity is more than ten-fold } \\
\text { higher }(17.6 \% \text { versus } \\
1.5 \%) \text { and a mean of } \\
6.2 \text { fewer days without } \\
\text { mechanical ventilation } \\
\text { and vasopressor support }\end{array}$ & \\
\hline T-chol & Serum & Sepsis $=136$ & - & - & - & - & - & $\begin{array}{l}\downarrow T \text {-chol associated with } \\
\text { risk of death in septic } \\
\text { patients } p<0.05\end{array}$ & [102] \\
\hline
\end{tabular}

Ang-1 angiopoietin-1, Ang-2 angiopoietin-2, APACHE-Il acute physiology and chronic health evaluation II, ARDS acute respiratory distress syndrome, $A U C$ area under the curve, $B B B$ blood-brain barrier, BMVEC brain microvascular endothelial cells, CD cluster of differentiation, CLDN-5 claudin-5, CRPC reactive protein, CSF cerebrospinal fluid, $D A M P s$ damage-associated molecular patterns, DIC disseminated intravascular coagulation, EEG electroencephalography, GCS Glasgow coma scale, $H D L$ high-density lipoprotein, HLA-DR human leukocyte antigen, HMGB1 high mobility group box 1, hsCRP high-sensitivity $C$ reactive protein, $I-F A B P$ intestinal fatty acid binding protein, $I L$ interleukin, $L D L$ low-density lipoprotein, Inc-ANRIL long non-coding antisense non-coding RNA in the INK4 locus, Inc-MALAT1 long non-coding metastasis-associated lung adenocarcinoma transcript 1, Inc-MEG3 long non-coding RNA maternally expressed gene 3, IncRNA long non-coding RNA, MCP-1 monocyte chemoattractant protein-1, miR-125a micro RNA-125a, miR-125b micro RNA-125b, MODS multiple organ dysfunction syndrome, MOF multiple organ failure, MPO myeloperoxidase, MR-proADM midregional pro adrenomedullin, NFL neurofilament light, $N f H$ neurofilament heavy, NSE neuron specific enolase, NT-proBNP N-terminal pro-brain natriuretic peptide, OCLN occludin, OR odds ratio, PAl-1 plasminogen activator inhibitor 1, PCT procalcitonin, $P T X-3$ pentraxin-3, RNA ribonucleic acid, S100B calcium-binding protein B, SE-Selectin soluble E-selectin, sFlt-1 soluble fms-like tyrosine kinase 1, sICAM-1 soluble intercellular adhesion molecule 1, SIRS systemic inflammatory response syndrome, SOFA sequential organ failure assessment, $S P D-1$ soluble programmed death protein 1, SPD-L1 soluble programmed death ligand 1, SPPB short physical performance battery, sTNFR1 soluble tumor necrosis factor receptor type 1, sTNFR2 soluble tumor necrosis factor receptor type 2, sTREM-1 soluble triggering receptor expressed on myeloid cells 1, suPAR soluble form of the urokinase plasminogen activator receptor, sVCAM-1 soluble vascular cell adhesion molecule 1, T-chol total cholesterol, TNF- $a$ tumour necrosis factor alpha, TREM-1 triggering receptor expressed on myeloid cells-1, VLA-3/a3 $\beta 1$ integrin alpha 3 beta 1, ZO-1 zonula-occluden 1). $\uparrow$ increase, $\downarrow$ decrease, $\leftrightarrow$ no difference 


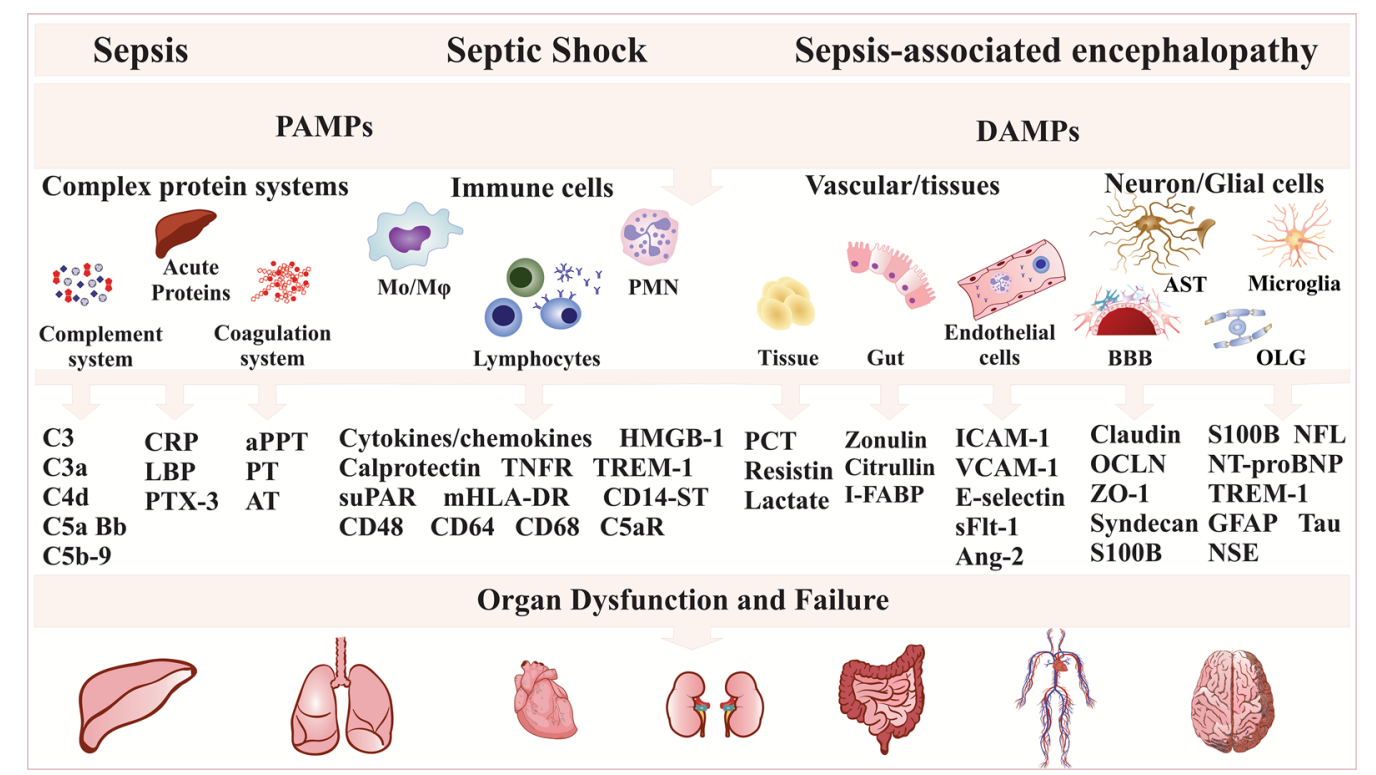

Fig. 1 Sepsis, septic shock, and sepsis-associated encephalopathy biomarkers. The infection triggers a cascade of signaling pathways that activate several transcription factors and promote proinflammatory mediators such as acute-phase proteins, cytokines, chemokines, and antimicrobial peptides necessary to eliminate the invading pathogens. The unbalanced host immune response triggers vascular endothelial damage, increasing gut and BBB permeability, culminating in organ dysfunction. Ang-2 (angiopoietin-2), APP (acute phase proteins), aPPT (activated partial thromboplastin), AST (astrocytes), AT (antithrombin), BBB (blood-brain barrier), C5aR (complement component 5a receptor), CD (cluster of differentiation), CD14-ST (soluble subtype of CD14), CRP (C reactive protein), DAMPs (damage-associated molecular patterns), GFAP (glial fibrillary acidic protein), HMGB-1 (high mobility group box 1), ICAM-1 (intercellular adhesion molecule 1), I-FABP (intestinal fatty acid binding protein), LBP (lipopolysaccharide binding protein), mHLA-DR (monocytic human leukocyte antigen DR), Mo (macrophage), NFL (neurofilament light), NSE (neuron specific enolase), NT-proBNP (N-terminal pro-brain natriuretic peptide), OCLN (occludin), OLG (oligodendrocyte), PAMPS (pathogen-associated molecular patterns), PCT (procalcitonin), PMNL (polymorphonuclear leukocytes), PT (prothrombin), PTX-3 (pentraxin-3), S100B (calcium-binding protein B), sFlt-1 (soluble fms-like tyrosine kinase 1), suPAR (soluble form of the urokinase plasminogen activator receptor), TNFR (tumor necrosis factor receptor type), TREM-1 (triggering receptor expressed on myeloid cells 1), VCAM-1 (vascular cell adhesion molecule 1), ZO-1 (zonula-occluden 1)

\section{Conclusion}

Despite significant advances in treating septic patients, this disease continues to be associated with high mortality rates and high long-term cognitive dysfunction. Extensive research in the area is being performed to validate biomarkers, facilitate sepsis diagnosis, and allow an early intervention that, although primarily supportive, can reduce the risk of death. Sepsis sometimes shows a hyperinflammatory response pattern and may be followed by an immunosuppressive phase, during which multiple organ dysfunction is present. A biomarker or a panel of biomarkers could be a new avenue to predict, identify, or provide new approaches to treat sepsis.

\section{Acknowledgements}

This work was supported by the Faillace Department of Psychiatry and Behavioral Sciences, McGovern Medical School, The University of Texas Health Science Center at Houston (UTHealth), USA (T.B.); the Graduate Program in Health Sciences, University of Southern Santa Catarina (UNESC) (T.B., JSG, and FDP), Brazil; the Alzheimer's Association and U.S. National Institute of Health/ National Institute on Aging (NIH/NIA (T.B.).

\begin{abstract}
Authors' contributions
Conceptualization: TB, MS, and FD-P; Writing —original draft: TB and JSG. Writing (review and editing): TB, JSG, MS, and FD-P. All authors read and approved the final manuscript.

\section{Funding}

The Alzheimer's Association Grant number AARGDNTF-19-619645 and U.S. National Institute of Health/National Institute on Aging ( $\mathrm{NIH} / \mathrm{NIA}$ Grant (1RF1AG072491-01) (T.B.); MCTIC/CNPq/FNDCT/MS/SCTIE/Decit No 401263/2020-7 (FD-P)).
\end{abstract}

\section{Availability of data and material \\ Not applicable.}

\section{Declarations}

Ethics approval and consent to participate

Not applicable.

\section{Consent for publication}

Not applicable.

\section{Competing interests}

The authors declare that they have no competing interests. 


\begin{abstract}
Author details
${ }^{1}$ Laboratory of Experimental Pathophysiology, Graduate Program in Health Sciences, University of Southern Santa Catarina (UNESC), Criciúma, SC, Brazil.

2Faillace Department of Psychiatry and Behavioral Sciences, McGovern Medical School, The University of Texas Health Science Center at Houston (UTHealth), Houston, TX 77054, USA. ${ }^{3}$ Bloomsbury Institute of Intensive Care Medicine, Division of Medicine, University College London, London, UK.
\end{abstract}

Received: 15 September 2021 Accepted: 8 December 2021 Published online: 06 January 2022

\section{References}

1. Arina P, Singer M. Pathophysiology of sepsis. Curr Opin Anaesthesiol. 2021;34(2):77-84.

2. Chakraborty RK, Burns B. Systemic inflammatory response syndrome. In: StatPearls. Treasure Island (FL): StatPearls Publishing. Copyright (c) 2021 StatPearls Publishing LLC:; 2021.

3. Vincent JL, Martin GS, Levy MM. qSOFA does not replace SIRS in the definition of sepsis. Crit Care (Lond, Engl). 2016;20(1):210.

4. Yende S, Kellum JA, Talisa VB, Peck Palmer OM, Chang CH, Filbin MR, Shapiro NI, Hou PC, Venkat A, LoVecchio F, et al. Long-term host immune response trajectories among hospitalized patients with sepsis. JAMA Netw Open. 2019;2(8):e198686.

5. Barichello T, Generoso JS, Dominguini D, Córneo E, Giridharan WV, Sahrapour TA, Simões LR, Rosa MID, Petronilho F, Ritter C, et al. Postmortem evidence of brain inflammatory markers and injury in septic patients: a systematic review. Crit Care Med. 2021. https://doi.org/10.1097/CCM. 0000000000005307

6. Kumar S, Ingle H, Prasad DV, Kumar H. Recognition of bacterial infection by innate immune sensors. Crit Rev Microbiol. 2013;39(3):229-46.

7. Heckenberg SG, Brouwer MC, van de Beek D. Bacterial meningitis. Handb Clin Neurol. 2014;121:1361-75.

8. Sellner J, Täuber MG, Leib SL. Pathogenesis and pathophysiology of bacterial CNS infections. In: Karen LR, Allan RT, editors. Handbook of clinical neurology, vol. 96. Amsterdam: Elsevier; 2010. p. 1-16.

9. Mook-Kanamori BB, Geldhoff M, van der Poll T, van de Beek D. Pathogenesis and pathophysiology of pneumococcal meningitis. Clin Microbiol Rev. 2011:24(3):557-91.

10. Gong T, Liu L, Jiang W, Zhou R. DAMP-sensing receptors in sterile inflammation and inflammatory diseases. Nat Rev Immunol. 2020;20(2):95-112. https://doi.org/10.1038/s41577-019-0215-7.

11. Iwasaki A, Medzhitov R. Regulation of adaptive immunity by the innate immune system. Science (New York, NY). 2010;327(5963):291-5.

12. Wiersinga WJ, Leopold SJ, Cranendonk DR, van der Poll T. Host innate immune responses to sepsis. Virulence. 2014;5(1):36-44.

13. Lu B, Wang C, Wang M, Li W, Chen F, Tracey KJ, Wang H. Molecular mechanism and therapeutic modulation of high mobility group box 1 release and action: an updated review. Expert Rev Clin Immunol. 2014:10(6):713-27.

14. Kigerl KA, de Rivero Vaccari JP, Dietrich WD, Popovich PG, Keane RW. Pattern recognition receptors and central nervous system repair. Exp Neurol. 2014;258:5-16.

15. Kataoka H, Kono H, Patel Z, Rock KL. Evaluation of the contribution of multiple DAMPs and DAMP receptors in cell death-induced sterile inflammatory responses. PLOS ONE. 2014:9(8):e104741.

16. Generoso JS, Giridharan WV, Lee J, Macedo D, Barichello T. The role of the microbiota-gut-brain axis in neuropsychiatric disorders. Braz J Psychiatry. 2020;10(44462020005021200):1516-4446.

17. Annane D, Sharshar T. Cognitive decline after sepsis. Lancet Respir Med. 2015;3(1):61-9.

18. Barichello T, Sayana P, Giridharan WV, Arumanayagam AS, Narendran B, Della Giustina A, Petronilho F, Quevedo J, Dal-Pizzol F. Long-term cognitive outcomes after sepsis: a translational systematic review. Mol Neurobiol. 2019:56(1):186-251.

19. Erreni M, Manfredi AA, Garlanda C, Mantovani A, Rovere-Querini P. The long pentraxin PTX3: a prototypical sensor of tissue injury and a regulator of homeostasis. Immunol Rev. 2017;280(1):112-25.

20. McFadyen JD, Zeller J, Potempa LA, Pietersz GA, Eisenhardt SU, Peter K. C-Reactive protein and its structural isoforms: an evolutionary conserved marker and central player in inflammatory diseases and beyond. Subcell Biochem. 2020;94:499-520.

21. Pierrakos C, Velissaris D, Bisdorff M, Marshall JC, Vincent JL. Biomarkers of sepsis: time for a reappraisal. Crit Care (Lond, Engl). 2020;24(1):287.

22. Yende S, Kellum JA, Talisa VB, Peck Palmer OM, Chang CH, Filbin MR, Shapiro NI, Hou PC, Venkat A, LoVecchio F, et al. Long-term host immune response trajectories among hospitalized patients with sepsis. JAMA Netw Open. 2019;2(8):e198686.

23. Caironi P, Masson S, Mauri T, Bottazzi B, Leone R, Magnoli M, Barlera S, Mamprin F, Fedele A, Mantovani A, et al. Pentraxin 3 in patients with severe sepsis or shock: the ALBIOS trial. Eur J Clin Invest. 2017:47(1):73-83.

24. Song J, Moon S, Park DW, Cho HJ, Kim JY, Park J, Cha JH. Biomarker combination and SOFA score for the prediction of mortality in sepsis and septic shock: a prospective observational study according to the Sepsis-3 definitions. Medicine. 2020:99(22):e20495.

25. Younger JG, Bracho DO, Chung-Esaki HM, Lee M, Rana GK, Sen A, Jones AE. Complement activation in emergency department patients with severe sepsis. Acad Emerg Med Off J Soc Acad Emerg Med. 2010;17(4):353-9.

26. Abe T, Kubo K, Izumoto S, Shimazu S, Goan A, Tanaka T, Koroki T, Saito K, Kawana R, Ochiai H. Complement activation in human sepsis is related to sepsis-induced disseminated intravascular coagulation. Shock (Augusta, Ga). 2020;54(2):198-204.

27. Matsumoto H, Ogura H, Shimizu K, Ikeda M, Hirose T, Matsuura H, Kang S, Takahashi K, Tanaka T, Shimazu T. The clinical importance of a cytokine network in the acute phase of sepsis. Sci Rep. 2018;8(1):13995.

28. Larsson A, Tydén J, Johansson J, Lipcsey M, Bergquist M, Kultima K, Mandic-Havelka A. Calprotectin is superior to procalcitonin as a sepsis marker and predictor of 30-day mortality in intensive care patients. Scand J Clin Lab Invest. 2020:80(2):156-61.

29. Matsuura R, Komaru Y, Miyamoto Y, Yoshida T, Yoshimoto K, Hamasaki Y, Nangaku M, Doi K. Different biomarker kinetics in critically ill patients with high lactate levels. Diagnostics (Basel, Switzerland). 2020;10(7):454.

30. Barichello T, Generoso JS, Collodel A, Petronilho F, Dal-Pizzol F. The blood-brain barrier dysfunction in sepsis. Tissue Barriers. 2021;9(1):1840912.

31. Zhao GJ, Li D, Zhao Q, Lian J, Hu TT, Hong GL, Yao YM, Lu ZQ. Prognostic value of plasma tight-junction proteins for sepsis in emergency department: an observational study. Shock. 2016;45(3):326-32.

32. Erikson K, Tuominen $H$, Vakkala M, Liisanantti JH, Karttunen T, Syrjälä $\mathrm{H}$, Ala-Kokko TI. Brain tight junction protein expression in sepsis in an autopsy series. Crit Care. 2020;24(1):385.

33. Skibsted S, Jones AE, Puskarich MA, Arnold R, Sherwin R, Trzeciak S, Schuetz P, Aird WC, Shapiro NI. Biomarkers of endothelial cell activation in early sepsis. Shock. 2013;39(5):427-32.

34. Ikeda M, Matsumoto H, Ogura H, Hirose T, Shimizu K, Yamamoto K, Maruyama I, Shimazu T. Circulating syndecan-1 predicts the development of disseminated intravascular coagulation in patients with sepsis. J Crit Care. 2018;43:48-53.

35. Bloomfield SM, McKinney J, Smith L, Brisman J. Reliability of S100B in predicting severity of central nervous system injury. Neurocrit Care. 2007:6(2):121-38

36. Wu L, Feng Q, Ai ML, Deng SY, Liu ZY, Huang L, Ai YH, Zhang L. The dynamic change of serum S100B levels from day 1 to day 3 is more associated with sepsis-associated encephalopathy. Sci Rep. 2020;10(1):7718.

37. Erikson K, Ala-Kokko TI, Koskenkari J, Liisanantti JH, Kamakura R, Herzig KH, Syrjälä H. Elevated serum S-100 $\beta$ in patients with septic shock is associated with delirium. Acta Anaesthesiol Scand. 2019;63(1):69-73

38. Assimakopoulos SF, Triantos C, Thomopoulos K, Fligou F, Maroulis I, Marangos M, Gogos CA. Gut-origin sepsis in the critically ill patient: pathophysiology and treatment. Infection. 2018:46(6):751-60.

39. Klaus DA, Motal MC, Burger-Klepp U, Marschalek C, Schmidt EM, Lebherz-Eichinger D, Krenn CG, Roth GA. Increased plasma zonulin in patients with sepsis. Biochem Med (Zagreb). 2013;23(1):107-11.

40. Zhang $X$, Liu D, Wang Y, Yan J, Yang X. Clinical significance on serum intestinal fatty acid binding protein and D-lactic acid levels in early intestinal injury of patients with sepsis. Zhonghua Wei Zhong Bing Ji Jiu Yi Xue. 2019:31(5):545-50 
41. Liu W, Geng F, Yu L. Long non-coding RNA MALAT1/microRNA 125a axis presents excellent value in discriminating sepsis patients and exhibits positive association with general disease severity, organ injury, inflammation level, and mortality in sepsis patients. J Clin Lab Anal. 2020;34(6):e23222.

42. Huang $X$, Zhao M. High expression of long non-coding RNA MALAT1 correlates with raised acute respiratory distress syndrome risk, disease severity, and increased mortality in sepstic patients. Int J Clin Exp Pathol. 2019;12(5):1877-87.

43. Na L, Ding H, Xing E, Gao J, Liu B, Wang H, Yu J, Yu C. Lnc-MEG3 acts as a potential biomarker for predicting increased disease risk, systemic inflammation, disease severity, and poor prognosis of sepsis via interacting with miR-21. J Clin Lab Anal. 2020;34(4):e23123.

44. Zhao D, Li S, Cui J, Wang L, Ma X, Li Y. Plasma miR-125a and miR-125b in sepsis: correlation with disease risk, inflammation, severity, and prognosis. J Clin Lab Anal. 2020;34(2):e23036.

45. Mahmoodpoor A, Movassaghpour A, Talebi M, Shadvar K, Soleimanpour $\mathrm{H}$. Value of flow cytometry (HLA-DR, CD14, CD25, CD13, CD64) in prediction of prognosis in critically ill septic patients admitted to ICU: a pilot study. J Clin Anesth. 2020;61:109646.

46. Yin WP, Li JB, Zheng XF, An L, Shao H, Li CS. Effect of neutrophil CD64 for diagnosing sepsis in emergency department. World J Emerg Med. 2020;11(2):79-86

47. Kondo Y, Umemura Y, Hayashida K, Hara Y, Aihara M, Yamakawa K. Diagnostic value of procalcitonin and presepsin for sepsis in critically ill adult patients: a systematic review and meta-analysis. J Intensive Care. 2019;7:22.

48. Lu B, Zhang Y, Li C, Liu C, Yao Y, Su M, Shou S. The utility of presepsin in diagnosis and risk stratification for the emergency patients with sepsis. Am J Emerg Med. 2018;36(8):1341-5.

49. Sen $S$, Kamit F, İşüder R, Yazıcı P, Bal Z, Devrim İ, Bayram SN, Karapınar B, Anıl AB, Vardar F. Surface TREM-1 as a Prognostic Biomarker in Pediatric Sepsis. Indian J Pediatr. 2020;88:134-40.

50. Andrés C, Andaluz-Ojeda D, Cicuendez R, Nogales L, Martín S, MartinFernandez M, Almansa R, Calvo D, Esteban-Velasco MC, Vaquero-Roncero LM, et al. MR- proADM to detect specific types of organ failure in infection. Eur J Clin Invest. 2020;50(6):19.

51. Schuetz P, Hausfater P, Amin D, Amin A, Haubitz S, Faessler L, Kutz A, Conca A, Reutlinger B, Canavaggio P, et al. Biomarkers from distinct biological pathways improve early risk stratification in medical emergency patients: the multinational, prospective, observational TRIAGE study. Crit Care. 2015;19(377):015-1098.

52. Elke G, Bloos F, Wilson DC, Brunkhorst FM, Briegel J, Reinhart K, Loeffler M, Kluge S, Nierhaus A, Jaschinski U, et al. The use of mid-regional proadrenomedullin to identify disease severity and treatment response to sepsis - a secondary analysis of a large randomised controlled trial. Crit Care (Lond, Engl). 2018;22(1):79.

53. Lundberg $\mathrm{OHM}$, Lengquist $M$, Spångfors $M$, Annborn M, Bergmann D, Schulte J, Levin H, Melander O, Frigyesi A, Friberg H. Circulating bioactive adrenomedullin as a marker of sepsis, septic shock and critical illness. Crit Care (Lond, Engl). 2020;24(1):636.

54. Honore PM, Redant S, De Bels D. Reliability of biomarkers of sepsis during extracorporeal therapies: the clinician needs to know what is eliminated and what is not. Crit Care (Lond, Engl). 2020;24(1):553.

55. Custodero C, Wu Q, Ghita GL, Anton SD, Brakenridge SC, Brumback BA, Efron PA, Gardner AK, Leeuwenburgh C, Moldawer LL, et al. Prognostic value of NT-proBNP levels in the acute phase of sepsis on lower longterm physical function and muscle strength in sepsis survivors. Crit Care. 2019;23(1):230.

56. Varpula M, Pulkki K, Karlsson S, Ruokonen E, Pettilä V. Predictive value of $\mathrm{N}$-terminal pro-brain natriuretic peptide in severe sepsis and septic shock. Crit Care Med. 2007;35(5):1277-83.

57. Mihajlovic D, Brkic S, Uvelin A, Draskovic B, Vrsajkov V. Use of presepsin and procalcitonin for prediction of SeptiFast results in critically ill patients. J Crit Care. 2017;40:197-201.

58. Leli C, Ferranti M, Marrano U, Al Dhahab ZS, Bozza S, Cenci E, Mencacci A. Diagnostic accuracy of presepsin (sCD14-ST) and procalcitonin for prediction of bacteraemia and bacterial DNAaemia in patients with suspected sepsis. J Med Microbiol. 2016;65(8):713-9.

59. Klouche K, Cristol JP, Devin J, Gilles V, Kuster N, Larcher R, Amigues L, Corne $\mathrm{P}$, Jonquet $\mathrm{O}$, Dupuy AM. Diagnostic and prognostic value of soluble CD14 subtype (Presepsin) for sepsis and community-acquired pneumonia in ICU patients. Ann Intensive Care. 2016;6(1):59.

60. Enguix-Armada A, Escobar-Conesa R. García-De La Torre A, De La Torre-Prados MV. Usefulness of several biomarkers in the management of septic patients: C-reactive protein, procalcitonin, presepsin and midregional pro-adrenomedullin. Clin Chem Lab Med. 2016;54(1):163-8.

61. Bloos F, Trips E, Nierhaus A, Briegel J, Heyland DK, Jaschinski U, Moerer O, Weyland A, Marx G, Gründling M, et al. Effect of sodium selenite administration and procalcitonin-guided therapy on mortality in patients with severe sepsis or septic shock: a randomized clinical trial. JAMA Intern Med. 2016;176(9):1266-76.

62. Bonaventura A, Carbone F, Vecchié A, Meessen J, Ferraris S, Beck E, Keim R, Minetti S, Elia E, Ferrara D, et al. The role of resistin and myeloperoxidase in severe sepsis and septic shock: results from the ALBIOS trial. Eur J Clin Investig. 2020;50:e1333.

63. Cao C, Gu J, Zhang J. Soluble triggering receptor expressed on myeloid cell-1 (sTREM-1): a potential biomarker for the diagnosis of infectious diseases. Front Med. 2017;11(2):169-77.

64. Wright SW, Lovelace-Macon L, Hantrakun V, Rudd KE, Teparrukkul P, Kosamo S, Liles WC, Limmathurotsakul D, West TE. sTREM-1 predicts mortality in hospitalized patients with infection in a tropical, middleincome country. BMC Med. 2020;18(1):159.

65. Anderson BJ, Calfee CS, Liu KD, Reilly JP, Kangelaris KN, Shashaty MGS, Lazaar AL, Bayliffe Al, Gallop RJ, Miano TA, et al. Plasma sTNFR1 and IL8 for prognostic enrichment in sepsis trials: a prospective cohort study. Crit Care (Lond, Engl). 2019;23(1):400.

66. Casagranda I, Vendramin C, Callegari T, Vidali M, Calabresi A, Ferrandu G, Cervellin G, Cavazza M, Lippi G, Zanotti I, et al. Usefulness of suPAR in the risk stratification of patients with sepsis admitted to the emergency department. Intern Emerg Med. 2015;10(6):725-30.

67. Sharma A, Ray S, Mamidipalli R, Kakar A, Chugh P, Jain R, Ghalaut MS, Choudhury S. A comparative study of the diagnostic and prognostic utility of soluble urokinase-type plasminogen activator receptor and procalcitonin in patients with sepsis and systemic inflammation response syndrome. Indian J Crit Care Med Peer Review Off Publ Indian Soc Crit Care Med. 2020;24(4):245-51.

68. Liu M, Zhang X, Chen H, Wang G, Zhang J, Dong P, Liu Y, An S, Wang L. Serum SPD-L1, upregulated in sepsis, may reflect disease severity and clinical outcomes in septic patients. Scand J Immunol. 2017:85(1):66-72

69. Yeh CF, Wu CC, Liu SH, Chen KF. Comparison of the accuracy of neutrophil CD64, procalcitonin, and C-reactive protein for sepsis identification: a systematic review and meta-analysis. Ann Intensive Care. 2019;9(1):5.

70. Zhang Y, Feng Q, Zhou S, Chen H. Downregulation of serum survivin correlates with increased inflammation, enhanced disease severity and worse prognosis in sepsis patients. Medicine. 2020;99(28):0000000000020272.

71. Hoppensteadt D, Tsuruta K, Hirman J, Kaul I, Osawa Y, Fareed J. Dysregulation of inflammatory and hemostatic markers in sepsis and suspected disseminated intravascular coagulation. Clin Appl Thromb Hemost. 2015;21(2):120-7.

72. Hamed S, Behnes M, Pauly D, Lepiorz D, Barre M, Becher T, Lang S, Akin I, Borggrefe M, Bertsch T, et al. Diagnostic value of Pentraxin-3 in patients with sepsis and septic shock in accordance with latest sepsis-3 definitions. BMC Infect Dis. 2017;17(1):554

73. Tian R, Wang X, Pan T, Li R, Wang J, Liu Z, Chen E, Mao E, Tan R, Chen Y, et al. Plasma PTX3, MCP1 and Ang2 are early biomarkers to evaluate the severity of sepsis and septic shock. Scand J Immunol. 2019;90(6):7.

74. Barre M, Behnes M, Hamed S, Pauly D, Lepiorz D, Lang S, Akin I, Borggrefe $M$, Bertsch T, Hoffmann U. Revisiting the prognostic value of monocyte chemotactic protein 1 and interleukin- 6 in the sepsis-3 era. J Crit Care. 2018:43:21-8.

75. Karlsson S, Pettilä V, Tenhunen J, Laru-Sompa R, Hynninen M, Ruokonen E. HMGB1 as a predictor of organ dysfunction and outcome in patients with severe sepsis. Intensive Care Med. 2008;34(6):1046-53.

76. Karakike E, Adami ME, Lada M, Gkavogianni T, Koutelidakis IM, Bauer M, Giamarellos-Bourboulis EJ, Tsangaris I. Late peaks of hmgb1 and sepsis outcome: evidence for synergy with chronic inflammatory disorders. Shock. 2019;52(3):334-9.

77. Lerman YV, Lim K, Hyun YM, Falkner KL, Yang H, Pietropaoli AP, Sonnenberg A, Sarangi PP, Kim M. Sepsis lethality via exacerbated tissue 
infiltration and TLR-induced cytokine production by neutrophils is integrin a3ß1-dependent. Blood. 2014;124(24):3515-23.

78. Sarangi PP, Hyun YM, Lerman YV, Pietropaoli AP, Kim M. Role of $\beta 1$ integrin in tissue homing of neutrophils during sepsis. Shock (Augusta, Ga). 2012;38(3):281-7.

79. Fang Y, Li C, Shao R, Yu H, Zhang Q. The role of biomarkers of endothelial activation in predicting morbidity and mortality in patients with severe sepsis and septic shock in intensive care: a prospective observational study. Thromb Res. 2018;171:149-54.

80. Yao B, Zhang LN, Ai YH, Liu ZY, Huang L. Serum S100 $\beta$ is a better biomarker than neuron-specific enolase for sepsis-associated encephalopathy and determining its prognosis: a prospective and observational study. Neurochem Res. 2014;39(7):1263-9.

81. Mikacenic C, Hahn WO, Price BL, Harju-Baker S, Katz R, Kain KC, Himmelfarb J, Liles WC, Wurfel MM. Biomarkers of endothelial activation are associated with poor outcome in critical illness. PLOS ONE. 2015;10(10):e0141251.

82. Crenn P, Neveux N, Chevret S, Jaffray P, Cynober L, Melchior JC, Annane D. Plasma L-citrulline concentrations and its relationship with inflammation at the onset of septic shock: a pilot study. J Crit Care. 2014;29(2):315.e311-316.

83. Ware LB, Magarik JA, Wickersham N, Cunningham G, Rice TW, Christman BW, Wheeler AP, Bernard GR, Summar ML. Low plasma citrulline levels are associated with acute respiratory distress syndrome in patients with severe sepsis. Crit Care. 2013;17(1):1-8.

84. Zhu X. MiR-125b but not miR-125a is upregulated and exhibits a trend to correlate with enhanced disease severity, inflammation, and increased mortality in sepsis patients. J Clin Lab Anal. 2020;34(3):e23094.

85. Gui F, Peng H, Liu Y. Elevated circulating Inc-ANRIL/miR-125a axis level predicts higher risk, more severe disease condition, and worse prognosis of sepsis. J Clin Lab Anal. 2019;33(6):e22917.

86. Westhoff D, Engelen-Lee JY, Hoogland ICM, Aronica EMA, van Westerloo DJ, van de Beek D, van Gool WA. Systemic infection and microglia activation: a prospective postmortem study in sepsis patients. Immun Ageing. 2019;16(18):019-0158

87. Ehler J, Petzold A, Wittstock M, Kolbaske S, Gloger M, Henschel J, Heslegrave A, Zetterberg H, Lunn MP, Rommer PS, et al. The prognostic value of neurofilament levels in patients with sepsis-associated encephalopathy - a prospective, pilot observational study. PLoS ONE. 2019;14(1):e0211184.

88. Anderson BJ, Reilly JP, Shashaty MGS, Palakshappa JA, Wysoczanski A, Dunn TG, Kazi A, Tommasini A, Mikkelsen ME, Schweickert WD, et al. Admission plasma levels of the neuronal injury marker neuron-specific enolase are associated with mortality and delirium in sepsis. J Crit Care. 2016:36:18-23.

89. Şen S, Kamit F, İ̧güder R, Yazııı P, Bal Z, Devrim I, Bayram SN, Karapınar B, AnIl AB, Vardar F. Surface TREM-1 as a Prognostic Biomarker in Pediatric Sepsis. Indian J Pediatr. 2020;23(10):020-03355.

90. Aksaray S, Alagoz P, Inan A, Cevan S, Ozgultekin A. Diagnostic value of STREM-1 and procalcitonin levels in the early diagnosis of sepsis. North Clin Istanb. 2016;3(3):175-82.

91. Brenner T, Uhle F, Fleming T, Wieland M, Schmoch T, Schmitt F, Schmidt K, Zivkovic AR, Bruckner T, Weigand MA, et al. Soluble TREM-1 as a diagnostic and prognostic biomarker in patients with septic shock: an observational clinical study. Biomark Biochem Ind Expos Response Susceptibility Chem. 2017;22(1):63-9.

92. Spoto S, Cella E, de Cesaris M, Locorriere L, Mazzaroppi S, Nobile E, Lanotte AM, Pedicino L, Fogolari M, Costantino S, et al. Procalcitonin and MR-proadrenomedullin combination with SOFA and qSOFA Scores for Sepsis diagnosis and prognosis: a diagnostic algorithm. Shock (Augusta, Ga). 2018;50(1):44-52.

93. Yang Y, Liang S, Geng J, Wang Q, Wang P, Cao Y, Li R, Gao G, Li L. Development of a nomogram to predict 30-day mortality of patients with sepsis-associated encephalopathy: a retrospective cohort study. J Intensive Care. 2020;8(45):020-00459.

94. Bonaventura A, Carbone F, Vecchié A, Meessen J, Ferraris S, Beck E, Keim $\mathrm{R}$, Minetti S, Elia E, Ferrara D, et al. The role of resistin and myeloperoxidase in severe sepsis and septic shock: Results from the ALBIOS trial. Eur J Clin Invest. 2020;50(10):13.
95. Maruchi Y, Tsuda M, Mori H, Takenaka N, Gocho T, Huq MA, Takeyama N. Plasma myeloperoxidase-conjugated DNA level predicts outcomes and organ dysfunction in patients with septic shock. Crit Care. 2018;22(1):018-2109.

96. Karampela I, Christodoulatos GS, Kandri E, Antonakos G, Vogiatzakis E, Dimopoulos G, Armaganidis A, Dalamaga M. Circulating eNampt and resistin as a proinflammatory duet predicting independently mortality in critically ill patients with sepsis: a prospective observational study. Cytokine. 2019;119:62-70.

97. Saboktakin L, Bilan N, Ghalehgolab Behbahan A, Poorebrahim S. Relationship between resistin levels and sepsis among children under 12 years of age: a case control study. Front Pediatr. 2019;7:355.

98. Ritter C, Tomasi CD, Dal-Pizzol F, Pinto BB, Dyson A, de Miranda AS, Comim CM, Soares M, Teixeira AL, Quevedo J, et al. Inflammation biomarkers and delirium in critically ill patients. Crit Care. 2014;18(3):1-6.

99. Feng Q, Wei WQ, Chaugai S, Leon BGC, Mosley JD, Leon DAC, Jiang $L$, Ihegword A, Shaffer CM, Linton MF, et al. Association between low-density lipoprotein cholesterol levels and risk for sepsis among patients admitted to the hospital with infection. JAMA Netw Open. 2019;2(1):e187223.

100. Chien JY, Jerng JS, Yu CJ, Yang PC. Low serum level of high-density lipoprotein cholesterol is a poor prognostic factor for severe sepsis. Crit Care Med. 2005;33(8):1688-93.

101. Cirstea M, Walley KR, Russell JA, Brunham LR, Genga KR, Boyd JH. Decreased high-density lipoprotein cholesterol level is an early prognostic marker for organ dysfunction and death in patients with suspected sepsis. J Crit Care. 2017;38:289-94.

102. Takegawa R, Kabata D, Shimizu K, Hisano S, Ogura H, Shintani A, Shimazu T. Serum albumin as a risk factor for death in patients with prolonged sepsis: an observational study. J Crit Care. 2019;51:139-44.

103. Sui YD, Xin WN, Feng LL. Comparison of the clinical application values of PCT, hs-CRP and SAA detection in the early diagnosis of sepsis. Pak J Med Sci. 2020;36(7):1683-7.

104. Hou X, Liu C, Lian H, Xu Z, Ma L, Zang X, Sun J, Jia K, Cui L. The value of neutrophil gelatinase-associated lipocalin and citrullinated alpha enolase peptide-1 antibody in diagnosis, classification, and prognosis for patients with sepsis. Medicine. 2020;99(34):0000000000021893.

105. Zhang H, Wang X, Zhang Q, Xia Y, Liu D. Comparison of procalcitonin and high-sensitivity C-reactive protein for the diagnosis of sepsis and septic shock in the oldest old patients. BMC Geriatr. 2017; 17(1):017-0566.

106. Lin R, Hu H, Li L, Chen G, Luo L, Rao P. The potential of microRNA-126 in predicting disease risk, mortality of sepsis, and its correlation with inflammation and sepsis severity. J Clin Lab Anal. 2020;34(9):2.

107. Zeng Q, Wu J, Yang S. Circulating IncRNA ITSN1-2 is upregulated, and its high expression correlates with increased disease severity, elevated inflammation, and poor survival in sepsis patients. J Clin Lab Anal. 2019:33(4):25

108. Piazza O, Russo E, Cotena S, Esposito G, Tufano R. Elevated S100B levels do not correlate with the severity of encephalopathy during sepsis. Br J Anaesth. 2007:99(4):518-21.

109. Crenn P, Neveux N, Chevret S, Jaffray P, Cynober L, Melchior JC, Annane D. Plasma L-citrulline concentrations and its relationship with inflammation at the onset of septic shock: a pilot study. J Crit Care. 2014;29(2):26.

110. Chen J, He Y, Zhou L, Deng Y, Si L. Long non-coding RNA MALAT1 serves as an independent predictive biomarker for the diagnosis, severity and prognosis of patients with sepsis. Mol Med Rep. 2020;21(3):1365-73.

111. Zhao D, Li S, Cui J, Wang L, Ma X, Li Y. Plasma miR-125a and miR-125b in sepsis: correlation with disease risk, inflammation, severity, and prognosis. J Clin Lab Anal. 2020;34(2):23036.

112. Ehler J, Saller T, Wittstock M, Rommer PS, Chappell D, Zwissler B, Grossmann A, Richter G, Reuter DA, Nöldge-Schomburg G, et al. Diagnostic value of NT-proCNP compared to NSE and S100B in cerebrospinal fluid and plasma of patients with sepsis-associated encephalopathy. Neurosci Lett. 2019;692:167-73.

113. Aksaray S, Alagoz P, Inan A, Cevan S, Ozgultekin A. Diagnostic value of STREM- 1 and procalcitonin levels in the early diagnosis of sepsis. North Clin Istanb. 2017;3(3):175-82.

114. Assicot M, Gendrel D, Carsin H, Raymond J, Guilbaud J, Bohuon C. High serum procalcitonin concentrations in patients with sepsis and infection. Lancet (Lond, Engl). 1993;341(8844):515-8. 
115. Saboktakin L, Bilan N, Ghalehgolab Behbahan A, Poorebrahim S. Relationship between resistin levels and sepsis among children under 12 years of age: a case control study. Front Pediatr. 2019;7:355.

\section{Publisher's Note}

Springer Nature remains neutral with regard to jurisdictional claims in published maps and institutional affiliations.

- fast, convenient online submission

- thorough peer review by experienced researchers in your field

- rapid publication on acceptance

- support for research data, including large and complex data types

- gold Open Access which fosters wider collaboration and increased citations

- maximum visibility for your research: over $100 \mathrm{M}$ website views per year

At BMC, research is always in progress.

Learn more biomedcentral.com/submissions 